\title{
On the numerical computation of Nonlinear Normal Modes for reduced-order modelling of conservative vibratory systems
}

\author{
F. Blanc ${ }^{\mathrm{a}}$, C. Touzéa,,* J.-F. Mercier ${ }^{\mathrm{b}}$, K. Ege ${ }^{\mathrm{c}}$, A.-S. Bonnet Ben-Dhia ${ }^{\mathrm{b}}$ \\ ${ }^{a}$ Unité de Mécanique (UME), ENSTA-ParisTech, Chemin de la Hunière, 91761 Palaiseau Cedex, France \\ ${ }^{b}$ Laboratoire POEMS, UMR 7231 CNRS/ENSTA/INRIA, ENSTA-ParisTech, 828, Boulevard des Maréchaux, \\ 91762 Palaiseau Cedex, France
}

${ }^{c}$ Laboratoire Vibrations Acoustique (LVA), INSA de Lyon, 25bis avenue Jean Capelle, 69621 Villeurbanne Cedex, France

\begin{abstract}
Numerical computation of Nonlinear Normal Modes (NNMs) for conservative vibratory systems is addressed, with the aim of deriving accurate reduced-order models up to large amplitudes. A numerical method is developed, based on the center manifold approach for NNMs, which uses an interpretation of the equations as a transport problem, coupled to a periodicity condition for ensuring manifold's continuity. Systematic comparisons are drawn with other numerical methods, and especially with continuation of periodic orbits, taken as reference solutions. Three different mechanical systems, displaying peculiar characteristics allowing for a general view of the performance of the methods for vibratory systems, are selected. Numerical results show that invariant manifolds encounter folding points at large amplitude, generically (but not only) due to internal resonances. These folding points involve an intrinsic limitation to reduced-order models based on the center manifold and on the idea of a functional relationship between slave and master coordinates. Below that amplitude limit, numerical methods are able to produce reduced-order models allowing for a precise prediction of the backbone curve.
\end{abstract}

Keywords: nonlinear normal modes, numerical computation, nonlinear vibrations, reduced-order models, invariant manifold

\section{Introduction}

Since their first introduction in the 1960's in Rosenberg's work [1, 2], Nonlinear Normal Modes (NNMs) have been widely used in nonlinear vibration theory, with the purpose of identification $[3,4,5,6,7]$, model order reduction $[8,9,10,11,12,13,14,15,16,17$, 18], or understanding of new phenomena such as targeted energy transfer [19, 20, 21, 22, 23]. They have been first introduced for conservative systems and thus defined in the framework provided by Lyapunov's theorem [24], i.e. a family of periodic orbits, that persist in the vicinity of fixed points with the presence of nonlinearity. This definition has been used by Rosenberg and other subsequent investigators who retained this framework [25, 26, 27, 28]. However, a more general definition has emerged since the work by Shaw and Pierre [29], who used the framework

${ }^{*}$ Corresponding author

Email addresses: francois.blanc@ensta-paristech.fr (F. Blanc), cyril.touze@ensta-paristech.fr (C. Touzé), jean-francois.mercier@ensta-paristech.fr (J.-F. Mercier), kerem.ege@insa-lyon.fr (K. Ege), anne-sophie.bonnet-bendhia@ensta-paristech.fr (A.-S. Bonnet Ben-Dhia) 
provided by the center manifold theorem [30] in order to define a NNM as an invariant manifold in phase space, tangent at origin to the corresponding eigenmode. This definition allows to handle the cases of damped and gyroscopic systems. On the other hand, it restricts the NNMs to be located in the vicinity of the eigenmodes of the system (through the tangency constraint at origin), while Rosenberg's definition allows defining as NNMs all the periodic orbits existing in phase space. A third alternative definition is given by using the framework provided by the theorems by Poincaré and Poincaré-Dulac [31,32], the so-called theory of normal forms, and allows expressing the NNMs of conservative [33,13] as well as damped mechanical systems [34].

The retained definition gives rise to different classes of methods for the computation of NNMs. Most of the existing methods derive from analytical calculations, as a legacy of history since the very first developments have been realized at a time when computers were not as powerful as today. However, since the last decade, more and more numerical methods have been established in order to extend the amplitude limitations encountered by asymptotic methods. Roughly speaking, Rosenberg's definition leads naturally to continuation methods for periodic orbits, while using the center manifold theorem leads to consider a Partial Differential Equation (PDE) in phase space for constructing the NNM, that can be solved by one of the numerous method provided by applied mathematics (finite difference, Galerkin projection, ...). For normal form, the proof of Poincaré's theorem brings the key for constructing NNMs that is solely computable by asymptotic developments.

More precisely, for those who use the definition of NNMs as a family of periodic orbits, numerous analytical methods have been proposed based on the use of the synchronicity of the motion [2, 26, 35], the extremum points of the energies [27] or the fact that those orbits are geodesics of selected subspaces [36]. A special class of methods derives from the idea of using a non-smooth temporal transformation (NSTT), proposed by Pilipchuk [37, 38]. This approach transforms the problem of finding periodic orbits to a two-points nonlinear boundary value problem (NLBVP) where the period plays the role of a nonlinear eigenvalue. The NLBVP can be solved either analytically by regular perturbation series [39], or numerically with the help of shooting technique [40]. Concerning numerical methods, a first approach by Slater was to use shooting methods for periodic orbits [41]. The natural extension was to use the powerful methods proposed in the framework of continuation techniques [42] for numerically computing the family of periodic orbits and thus the NNMs, with no amplitude limitations [43, 44]. Continuation of periodic orbits has now been recognized as the most efficient method for conservative systems, since they are able to easily handle the presence of turning points, internal resonances, to compute the stability domains and to deal with numerous other difficulties that are specific to nonlinear systems. Moreover, their implementation is now well documented within numerous standard methods for steps continuation and adaptation (arclength, pseudo-arclength, asymptotic-numerical method). Finally, standard codes such as AUTO [45], MANLAB [46, 47] or MATCONT [48] can now be routinely used for the purpose of practical computations.

The center manifold theorem gives a constructive method relying on the definition of slave and master coordinates that are linked through an unknown function, which is solution of a PDE expressing the geometry of the invariant manifold in phase space [30, 29]. Solutions of this PDE has first been sought through asymptotic developments [8, 49, 3]. Then, numerical methods have been proposed, relying on Galerkin expansions on orthogonal polynomials $[11,50]$ or functions with compact supports mimicking finite elements $[5,51,52,53]$. Finally, 
finite differences coupled to a marching scheme for the amplitude, playing the role of time, has been proposed by Noreland et al [18]. Although they do not use formally the center manifold theorem, their amplitude-phase representation gives rise to equations that share common points with the former method.

From the literature review, it appears that for conservative systems, continuation methods are the most efficient and versatile numerical method to get a complete picture of the NNMs of a system. The most recent obtained results show the ability of these methods to handle complex systems with a large number of degrees of freedom [54], or complicated dynamics with numerous solution branches [40]. However, continuation methods are restricted by construction to conservative systems, and the presence of a light damping has already been assessed as having an influence on the performance of reduced-order models based on NNMs [34].

The objective of this paper is twofold. First to define a numerical method for computing the NNMs of a nonlinear mechanical system. As applications to damped systems are targeted, the invariant manifold approach is selected. A new interpretation of the PDE defining the NNM in terms of a transport equation is proposed, leading to a numerical method based on finite differences and optimization of a periodicity criterion on the unknown initial condition. This paper is restricted to conservative systems in order to compare our results to those obtained with continuation methods, taken as reference solutions. However, direct extensions of this work will consider the case of damped systems. The second objective is to use the computed NNMs in the context of reduced-order models (ROMs). For conservative systems, ROMs' performance will be judged on their ability to predict correctly the backbone curve of the selected NNMs. The validity limits of NNM-based ROMs will be properly studied for three different systems by carefully inspecting the behaviour of the invariant manifold at large amplitudes. We will show that generically, invariant manifolds observe foldings due to internal resonances. These folding points introduce a fundamental limitation to NNM-based ROMs constructed with the invariant manifold approach (whatever the selected numerical method is), as the initial assumption of a functional relationship between slave and master coordinates breaks down.

The paper is organized as follows. First, the numerical method is presented in section 2, and its performance is assessed on a two degrees-of-freedom (dof) system with cubic nonlinearity, already selected by Pesheck et al. Section 3 is devoted to reduced-order models construction, from the numerical computation obtained in section 2. The ability of the ROM to capture the backbone curve of the first example is discussed, then a second two dofs example with quadratic and cubic nonlinearities is also studied. Finally the whole procedure is applied to a continuous system in section 4: a linear beam resting on an elastic nonlinear foundation. Conclusions are drawn in section 5 .

\section{Numerical computation for NNMs}

Geometric nonlinearity is considered in this paper, so that the starting point of the study is an assembly of $N$ oscillator equations displaying quadratic and cubic nonlinear coupling terms, that are generic to describe thin structures vibrating at large amplitudes. Semi-discrete equations are also taken into account, which means that we assume that space discretization has already been realized if one deals with a continuous structure. In the conservative case, model equations thus reads: 


$$
\forall k=1, \ldots, N: \quad \ddot{\eta}_{k}+\omega_{k}^{2} \eta_{k}=f_{k}(\boldsymbol{\eta}),
$$

where $f_{k}(\boldsymbol{\eta})=\sum_{i=1}^{N} \sum_{j \geq i}^{N} g_{i j}^{k} \eta_{i} \eta_{j}+\sum_{i=1}^{N} \sum_{j \geq i}^{N} \sum_{l \geq j}^{N} h_{i j l}^{k} \eta_{i} \eta_{j} \eta_{l}$ merges the quadratic and cubic nonlinearities. Note that no linear coupling terms are assumed in (1), which means that the linear mode basis has been retained for space discretization, so that $\eta_{k}$ is the modal displacement of the $k^{\text {th }}$ mode. Furthermore, it is assumed that $f_{k}$ depends on $\boldsymbol{\eta}$ and not on $\dot{\boldsymbol{\eta}}$, as it is usual for thin structures with geometric nonlinearities. This assumption has been selected for convenience of the presentation and is not restrictive for the forthcoming developments.

\subsection{Invariant manifold equations}

The general methodology provided by the center manifold theorem $[30,55]$ is applied to derive the invariant manifold equations defining the NNMs, as proposed in [29]. Let $p$ be the label of the master coordinate that defines the NNM under study. As second-order problem (in time) are at hand in vibration theory, it is usual to set (1) into its first-order formulation by using the displacement $\eta_{k}$ and the velocity $\xi_{k}=\dot{\eta}_{k}$. Thus a pair of master coordinates $\left(\eta_{p}, \xi_{p}\right)$ is given and the first assumption is to define a functional relationship between all the pairs of slave coordinates $\left(\eta_{k}, \xi_{k}\right), k \neq p$ and the master one.

As a conservative problem is here considered, which means that regular solutions will be closed periodic orbits, it appears natural to use polar coordinates instead of a cartesian rectangular grid, as proposed in [50]. Hence the master coordinates are transformed to:

$$
\begin{aligned}
\eta_{p} & =a \cos \phi, \\
\xi_{p} & =-a \omega_{p} \sin \phi .
\end{aligned}
$$

The slave coordinates, for all $k \neq p$, are then sought as functions of $(a, \phi)$ via functional relationships:

$$
\begin{aligned}
& \eta_{k}=P_{k}(a, \phi), \\
& \xi_{k}=Q_{k}(a, \phi),
\end{aligned}
$$

where $\left(P_{k}, Q_{k}\right)$ are the unknowns to be found. Differentiating (3) with respect to time leads to the following equations:

$$
\begin{aligned}
& \dot{\eta}_{k}=\dot{a} \partial_{a} P_{k}+\dot{\phi} \partial_{\phi} P_{k}=Q_{k}, \\
& \dot{\xi}_{k}=\dot{a} \partial_{a} Q_{k}+\dot{\phi} \partial_{\phi} Q_{k}=f_{k}-\omega_{k}^{2} P_{k} .
\end{aligned}
$$

This set of equations can be rewritten in a simplified manner as:

$$
\begin{aligned}
& \mathbf{V} \cdot \nabla P_{k}=Q_{k}, \\
& \mathbf{V} \cdot \nabla Q_{k}=f_{k}-\omega_{k}^{2} P_{k},
\end{aligned}
$$

i.e. like a transport equation with nonlinear source terms, where the velocity vector $\mathbf{V}=(\dot{a}, \dot{\phi})$ is simply given by the flow of the master coordinate. Finally, the $p^{\text {th }}$ equation in (1) allows to express the dynamics of the master mode:

$$
\begin{aligned}
& \dot{a}=-\frac{f_{p} \sin \phi}{\omega_{p}}, \\
& \dot{\phi}=\omega_{p}-\frac{f_{p} \cos \phi}{a \omega_{p}} .
\end{aligned}
$$


Note that division by $a$ in (6b) is not a problem because $f_{p}$ vanishes when $a \rightarrow 0$, and behaves at least like $a^{2}$ from its definition together with the tangency condition imposed by the NNMs, so that $\dot{\phi}$ remains bounded when $a \rightarrow 0$.

Substituting for (6) in (4) leads to eliminate the time variable. The resulting 2(N-2) PDEs are then purely geometrical: they define the geometry of the invariant manifold (the $p^{\text {th }} \mathrm{NNM}$ ) in phase space. The next subsections deal with the numerical method proposed to solve these equations, based on their transport problem structure.

Figure 1 shows the flow of the master mode for the two dofs problem presented in section 2.6. The figure has been obtained once the numerical computation of the manifold is realized, with the methodology described below. When $a \longrightarrow 0$, the nonlinearities are negligible and closed orbits are circles so that in the retained polar coordinates a regular velocity field is at hand. For increasing values of $a$, nonlinearities distord the closed orbits, thus giving rise to an oscillating part of the flow when $\phi$ varies.

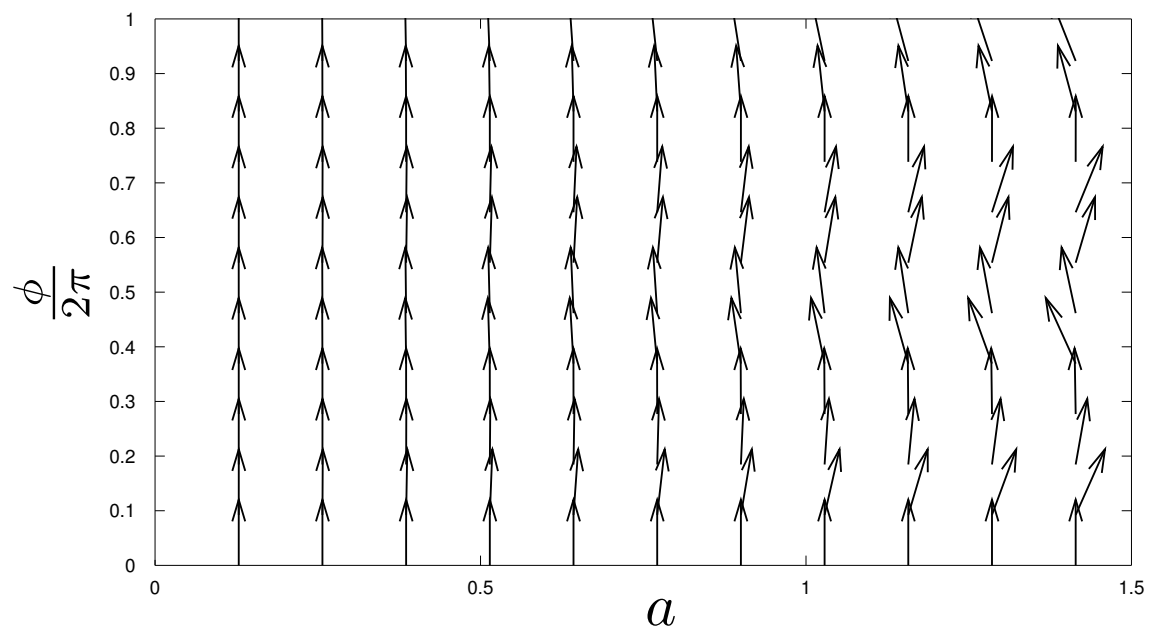

Figure 1: Flow of the master coordinates on the invariant manifold for the two dof problem with cubic nonlinearities shown in section 2.6 .

\subsection{The periodic problem to solve}

To simplify the presentation, we consider that the initial problem has two dofs, that the master mode is the first one, and the second is the slave, thus $p=1$ and $k=2$. Noting simply $P$ and $Q$ the unknowns $P_{2}$ and $Q_{2}$ we wish to determine, we have to solve the transport problem:

$$
\begin{aligned}
\vec{V} \cdot \vec{\nabla} P-Q & =0, \\
\vec{V} \cdot \vec{\nabla} Q+\omega_{2}^{2} P & =f_{2},
\end{aligned}
$$

with the velocity:

$$
\vec{V}=\left[\begin{array}{c}
V_{\phi} \\
V_{a}
\end{array}\right]=\left[\begin{array}{c}
\omega_{1}-\frac{f_{1} \cos \phi}{a \omega_{1}} \\
-\frac{f_{1} \sin \phi}{\omega_{1}} .
\end{array}\right] .
$$

Note that $f_{1}$ and $f_{2}$ are nonlinear functions of $\eta_{1}=a \cos \phi$ and $\eta_{2}=P(a, \phi)$. 
A usual way to solve this transport problem is to follow the characteristics of the flow. For small $a$-values, $V_{\phi} \simeq \omega_{1}$ and $V_{a} \simeq 0$ : the characteristics are the lines $a=c$ st oriented from $\phi=0$ to $\phi=2 \pi$. Therefore the problem reduces to a transport only along the $\phi$ direction and the solution has just to be specified at $\phi=0$ to be obtained everywhere. For increasing $a$-values the situation becomes more complicated and the characteristics are no longer straight lines. However, we have found in practice that $V_{\phi}>0$ everywhere and thus the characteristics still connect $\phi=0$ to $\phi=2 \pi$ (see Figure 1). Due to this general behaviour of the shape of the characteristics, we have chosen to treat the problem as a transport problem along the $\phi$ direction: solving the problem in the rectangle $(a, \phi) \in R=] 0, A[\times] 0,2 \pi$ [ where $A>0$ is fixed, we impose the solution at $\phi=0$, where the flux enters. Moreover, the solution must be periodic (continuity of the manifold), which is not the case for an arbitrary initial condition. An iterative procedure is then used to determine the initial condition such that a periodicity constraint is fulfilled. Let us point out that our approach is different from previous ones as $[50,18]$ where the other choice was retained: differential equations in the variable $a$ were solved while azimuthal decomposition on $e^{i n \phi}$ functions were used for the angle variable.

To close the problem, boundary conditions where the flux enters in the domain, have to be specified. At $\phi=0$, trial functions will be given. At $a=A$, and for $\phi$ values such that $V_{a}(A, \phi)<0$, the practical solution retained for the computation consists in extrapolating the manifold, assuming regularity. This point will be further detailed in section 2.5.

To summarize, for any $A>0$ values, the transport problem we propose to solve for $a \in] 0, A[$ and $\phi \in] 0,2 \pi[$ is:

The transport problem with periodicity constraint: for $P_{0}(a)$ and $Q_{0}(a)$ given functions, find $P(a, \phi)$ and $Q(a, \phi)$, solutions of (7), such that $P(a, 0)=$ $P_{0}(a), Q(a, 0)=Q_{0}(a)$ and $P(a, 2 \pi)=P_{0}(a), Q(a, 2 \pi)=Q_{0}(a)$, and assuming regularity at the boundary $a=A$ where $V_{a}(A, \phi)<0$.

This problem is approximated by a minimization problem:

The minimization problem: Find $P_{0}(a)$ and $Q_{0}(a)$ such that the cost functional:

$$
J\left(P_{0}, Q_{0}\right)=\frac{1}{2} \int_{0}^{A}\left(\left[P(a, 2 \pi)-P_{0}(a)\right]^{2}+\left[Q(a, 2 \pi)-Q_{0}(a)\right]^{2}\right) \mathrm{d} a,
$$

is minimum where $P(a, \phi)$ and $Q(a, \phi)$ are the solutions of the transport problem (7) for the initial conditions $P_{0}(a)$ and $Q_{0}(a)$.

To solve this minimization problem, we consider it as an iterative procedure where the new initial conditions at step $n+1$ are deduced from the initial conditions at step $n$ by following the direction of the gradient of $J$, which corresponds to the relationship:

$$
\left(\begin{array}{c}
P_{0}^{n+1} \\
Q_{0}^{n+1}
\end{array}\right)=\left(\begin{array}{c}
P_{0}^{n} \\
Q_{0}^{n}
\end{array}\right)-\theta_{n} \vec{\nabla} J\left(P_{0}^{n}, Q_{0}^{n}\right)
$$

where $\theta_{n}>0$ is a parameter that has to be suitably chosen in the numerical process and $\vec{\nabla} J$ is the gradient of the functional $J\left(P_{0}, Q_{0}\right)$. The task now is to determine this functional gradient, which is realized by using the method of the adjoint state [56]. 


\subsection{Gradient determination}

Two steps are necessary in order to derive the adjoint problem: first a linearization of the transport problem (7) with respect to small perturbation in the initial conditions, then taking the adjoint state of this linearized problem. For the sake of brevity, only the final result is given here. More details and complete demonstration can be found in Appendix A. The transport problem may be written as $\partial_{\phi} P \triangleq F_{P}, \partial_{\phi} Q \triangleq F_{Q}$, with:

$$
\begin{aligned}
& F_{P}=\frac{Q}{\omega_{1}}+\frac{f_{1}}{\omega_{1}^{2}}\left(\sin \phi \partial_{a} P+\frac{\cos \phi}{a} \partial_{\phi} P\right) \\
& F_{Q}=-\frac{\omega_{2}^{2} P}{\omega_{1}}+\frac{f_{1}}{\omega_{1}^{2}}\left(\sin \phi \partial_{a} Q+\frac{\cos \phi}{a} \partial_{\phi} Q\right)+\frac{f_{2}}{\omega_{1}} .
\end{aligned}
$$

Introducing the following functions so as to simplify the resulting equations:

$$
\begin{aligned}
& \alpha_{1}=\frac{\sin \phi}{\omega_{1}^{2}} \partial_{a} P \partial_{P} f_{1}+\frac{\cos \phi}{a \omega_{1}^{2}} \partial_{\phi} P \partial_{P} f_{1}, \\
& \alpha_{2}=\frac{f_{1} \sin \phi}{\omega_{1}^{2}}, \quad \alpha_{3}=\frac{f_{1} \cos \phi}{a \omega_{1}^{2}}
\end{aligned}
$$

the adjoint problem is expressed as: for $\tilde{p}_{2 \pi}(a)$ and $\tilde{q}_{2 \pi}(a)$ given functions, find $\tilde{p}(a, \phi)$ and $\tilde{q}(a, \phi)$ solutions of:

$$
-\partial_{\phi}\left(\begin{array}{c}
\tilde{p} \\
\tilde{q}
\end{array}\right)=\left[\begin{array}{cc}
\beta_{1}-\partial_{a}\left(\beta_{2}(.)\right) & \beta_{3} \partial_{P} F_{Q} \\
\beta_{3} \partial_{Q} F_{P} & -\partial_{a}\left(\beta_{2}(.)\right)
\end{array}\right]\left(\begin{array}{c}
\tilde{p} \\
\tilde{q}
\end{array}\right)
$$

with $\beta_{1}=\frac{\alpha_{1}}{1-\alpha_{3}}, \beta_{2}=\frac{\alpha_{2}}{1-\alpha_{3}}$ and $\beta_{3}=\frac{1}{1-\alpha_{3}}$. The end conditions $\tilde{p}_{2 \pi}(a)$ and $\tilde{q}_{2 \pi}(a)$ will be specified in the next paragraph. This adjoint problem has to be solved in the backward sense, from $\phi=2 \pi$ to 0 . Consequently, at $a=A$, the boundary condition has to be specified where the flux of the direct problem goes out (in a dual manner of the direct problem). In practical implementation, the same assumption of regularity and extrapolation will be used also for the adjoint problem.

As shown in Appendix A, the expression of the functional gradient can be easily determined from ad-hoc properties of the adjoint state, and reads:

$$
\vec{\nabla} J\left(P_{0}, Q_{0}\right)=\left[\begin{array}{c}
\tilde{p}(a, 0)-\tilde{p}_{2 \pi}(a) \\
\tilde{q}(a, 0)-\tilde{q}_{2 \pi}(a)
\end{array}\right],
$$

where the appropriate end condition (or initial condition with reverse time) for the adjoint problem has to be selected as:

$$
\begin{aligned}
& \tilde{p}_{2 \pi}(a)=P(a, 2 \pi)-P_{0}(a), \\
& \tilde{q}_{2 \pi}(a)=Q(a, 2 \pi)-Q_{0}(a) .
\end{aligned}
$$

Therefore the numerical evaluation of the functional gradient just requires to solve the adjoint problem from $\phi=2 \pi$ to 0 . We can now summarize the resolution algorithm.

\subsection{Algorithm}

Here we describe the stages of the algorithm. We suppose that $A$ is chosen. The step $n$ of the iterative minimization process is as follows: 
1. Solve the nonlinear transport problem (7) from the initial conditions $P_{0}^{n}(a)$ and $Q_{0}^{n}(a)$ to get $P^{n}(a, \phi)$ and $Q^{n}(a, \phi)$ in the rectangle $R$.

2. Solve the adjoint problem (12) from the end conditions:

$$
\begin{aligned}
& \tilde{p}_{2 \pi}^{n}(a)=P^{n}(a, 2 \pi)-P_{0}^{n}(a), \\
& \tilde{q}_{2 \pi}^{n}(a)=Q^{n}(a, 2 \pi)-P_{0}^{n}(a) .
\end{aligned}
$$

3. Determine $\vec{\nabla} J\left(P_{0}^{n}, Q_{0}^{n}\right)$ thanks to the formula (A.11) and define the new initial conditions $P_{0}^{n+1}$ and $Q_{0}^{n+1}$ by:

$$
\left(\begin{array}{c}
P_{0}^{n+1} \\
Q_{0}^{n+1}
\end{array}\right)=\left(\begin{array}{c}
P_{0}^{n} \\
Q_{0}^{n}
\end{array}\right)-\theta_{n} \vec{\nabla} J\left(P_{0}^{n}, Q_{0}^{n}\right)
$$

4. Solve the nonlinear transport problem (7) and evaluate $J\left(P_{0}^{n+1}, Q_{0}^{n+1}\right)$ from formula (8).

5. Test if $J\left(P_{0}^{n+1}, Q_{0}^{n+1}\right)<J\left(P_{0}^{n}, Q_{0}^{n}\right)$ :

- If yes, go to point 6

- If no, replace $\theta_{n}$ with $\theta_{n} / 2$ until $J\left(P_{0}^{n+1}, Q_{0}^{n+1}\right)<J\left(P_{0}^{n}, Q_{0}^{n}\right)$ and go to point 6

6. double the value of $\theta_{n}$.

To initiate the iterative process, we take for $P_{0}^{0}(a)$ and $Q_{0}^{0}(a)$ the asymptotic solution of the NNM, available e.g. from [13], or from the linear eigenmode solution, defined by $P_{k}=Q_{k} \equiv 0$. $\theta_{0}$ is taken as any small value, the doubling of $\theta_{n}$ at each step allowing to reach quickly nonsmall values. The iterations are stopped when $J$ becomes lower than a given tolerance value.

\subsection{The Finite Difference Numerical scheme}

A first-order finite difference scheme is selected to solve numerically the invariant manifold equations (7). In particular this allows to take easily into account the non linearities of the velocity and of the source term. Note that a Finite Element method would require to use an iterative process to deal with the non linearities, which would add to the iterative treatment of the periodicity we already use. A rectangular grid is introduced for computation: $0 \leq a_{m}=$ $m \Delta a \leq A$ for $m \in\left[0, N_{a}\right]$ and $0 \leq \phi_{n}=n \Delta \phi \leq 2 \pi$ for $n \in\left[0, N_{\phi}\right]$ with $N_{a} \Delta a=A$ and $N_{\phi} \Delta_{\phi}=2 \pi$. The unknowns $P(a, \phi)$ and $Q(a, \phi)$ are interpolated by their discrete values: $P_{n}^{m}=P\left(a_{m}, \phi_{n}\right)$ and $Q_{n}^{m}=Q\left(a_{m}, \phi_{n}\right)$. The transport problem (7) is solved for increasing values of $\phi$ starting from the initial conditions $\left(P_{0}(a), Q_{0}(a)\right)$ at $\phi=0$ and for $a \in[0, A]$. As it is standard for finite differences discretisation of transport equations, the choice of ( $a$-wise) backward/forward decentered scheme is dictated by the direction of the flow. This is done at the line $n+1$ according to the sign of $V_{a}(a, \phi)$ at the line $n$. Therefore we look for the sign of:

$$
V_{a}\left(a_{m}, \phi_{n}\right) \equiv V_{a}^{n, m}=-\frac{f_{1}^{n, m} \sin \phi_{n}}{\omega_{1}},
$$

where $f_{1}^{n, m}$ stands for the value of the nonlinear function $f_{1}$ evaluated at the current grid point $\left(a_{m}, \phi_{n}\right)$. Then the horizontal derivative $\partial_{a} P$ is approximated by

$$
\begin{aligned}
& \left(\frac{\partial P}{\partial a}\right)\left(a_{m}, \phi_{n}\right) \simeq \frac{P_{n}^{m}-P_{n}^{m-1}}{\Delta a} \quad \text { if } \quad V_{a}^{n, m}>0, \\
& \left(\frac{\partial P}{\partial a}\right)\left(a_{m}, \phi_{n}\right) \simeq \frac{P_{n}^{m+1}-P_{n}^{m}}{\Delta a} \quad \text { if } \quad V_{a}^{n, m}<0 .
\end{aligned}
$$


In practice we find that the sign of $V_{a}$ does not depend on $m$, it is constant along the line $n$ and we just take care of the sign of $V_{a}^{n, N_{a}}$ (in $a=A$ ).

Precisely the numerical scheme is the following: supposing the $n^{\text {th }}$ line is known, $n=0$ to $N_{\phi}-1$, the line $n+1$ is obtained thanks to the relations (given only for the $P$ unknown):

- If $V_{a}^{n, N_{a}}>0$ :

$$
\begin{array}{lll}
P_{n+1}^{m}=P_{n}^{m}+\frac{\Delta \phi}{\omega_{1}-\frac{f_{1}^{n, m} \cos \phi_{n}}{a_{m} \omega_{1}}}\left(Q_{n}^{m}+\frac{f_{1}^{n, m} \sin \phi_{n}}{\omega_{1}} \frac{P_{n}^{m}-P_{n}^{m-1}}{\Delta a}\right) & \text { for } & m=1 \text { to } N_{a}, \\
P_{n+1}^{0}=0 & \text { for } & m=0 .
\end{array}
$$

- If $V_{a}^{n, N_{a}}<0$ :

$$
P_{n+1}^{m}=P_{n}^{m}+\frac{\Delta \phi}{\omega_{1}-\frac{f_{1}^{n, m} \cos \phi_{n}}{a_{m} \omega_{1}}}\left(Q_{n}^{m}+\frac{f_{1}^{n, m} \sin \phi_{n}}{\omega_{1}} \frac{P_{n}^{m+1}-P_{n}^{m}}{\Delta a}\right) \quad \text { for } \quad m=N_{a} \quad \text { to } 0 .
$$

The scheme is similar for the $Q$ unknown. The assumption of manifold's regularity at the boundary $a=A$ where entering flux occurs (case $V_{a}^{n, N_{a}}<0$ ), allows now to extrapolate the missing value appearing in the FD scheme: once we know the $n^{\text {th }}$ line, a value $P_{n}^{N_{a}+1}$ outside the computation domain is determined by continuous extrapolation. Then the value $P_{n+1}^{N_{a}}$, which requires the point $P_{n}^{N_{a}+1}$ to be computed, can be evaluated.

\subsection{A system with cubic nonlinearities}

In this section, we consider a system presented by Pesheck et al. [50], consisting of two masses connected to nonlinear springs. This example is selected so as to compare our results (referred to as the "transport method" in the remainder of the paper) with those obtained by Pesheck et al. in [50], using Galerkin projection on a given functional basis for solving out the manifold equations. The equations of motion read:

$$
\left\{\begin{aligned}
m_{1} \ddot{q}_{1}+k_{1} q_{1}+k_{2}\left(q_{1}-q_{2}\right) & =-\gamma_{1} q_{1}^{3}-\gamma_{2}\left(q_{1}-q_{2}\right)^{3} \\
m_{2} \ddot{q}_{2}+k_{2}\left(q_{2}-q_{1}\right) & =-\gamma_{2}\left(q_{2}-q_{1}\right)^{3} .
\end{aligned}\right.
$$

The parameters values have been selected as in [50]: $m_{1}=m_{2}=1, k_{1}=1, k_{2}=5, \gamma_{1}=2$ and $\gamma_{2}=1$, so that the eigenfrequencies are equal to $\omega_{1}=0.689$ and $\omega_{2}=3.244$. With a linear transformation from the initial coordinates $\left(q_{1}, q_{2}\right)$ to the modal coordinates $\left(\eta_{1}, \eta_{2}\right)$, a problem of the form (1) is found with:

$$
\left\{\begin{array}{l}
f_{1}\left(\eta_{1}, \eta_{2}\right)=-0.405 \eta_{1}^{3}-1.34 \eta_{1}^{2} \eta_{2}-1.51 \eta_{1} \eta_{2}^{2}-0.349 \eta_{2}^{3} \\
f_{2}\left(\eta_{1}, \eta_{2}\right)=-0.448 \eta_{1}^{3}-1.51 \eta_{1}^{2} \eta_{2}-1.05 \eta_{1} \eta_{2}^{2}-4.58 \eta_{2}^{3}
\end{array}\right.
$$

Before showing the results given by the transport method for computing the NNMs, we first exhibit reference solutions obtained by continuation, so as to have a clear picture of the global dynamics of the system, from small to large amplitudes. Two different softwares have been used for continuation: AUTO (Predictor-corrector method with orthogonal collocation and pseudoarclength continuation) [45], and MANLAB (harmonic balance methods for periodic orbits and continuation of harmonics amplitudes with asymptotic-numerical method) [47, 46]. Figure 2 
shows the backbone curves for the two modes of the system, either represented with respect to energy (resulting in a so-called Frequency-Energy Plot, FEP), or with respect to amplitude of the master coordinate.

The system presents a globally hardening behaviour, as expected from the positive values of the selected coefficients $\left(\gamma_{1}, \gamma_{2}\right)$. While the second mode has a quite simple behaviour, the first mode exhibits a loop with a loss of stability. This behaviour is typical of the presence of a 1:3 internal resonance with the second mode, which is assessed by simply plotting (either on the FEP, or on the classical backbone curve) the backbone of the second mode where the frequency has been divided by three. The loop appears exactly when the ratio 1:3 is fulfilled. As already noted in [6], here a 1:3 internal resonance is observed on the nonlinear frequencies, as the linear eigenfrequencies do not show a ratio 1:3. The loop is also depicted in Fig. 2(b), where the amplitudes of the two modal coordinates $\left(\eta_{1}, \eta_{2}\right)$ have been represented. One can observe the sudden increase of $\eta_{2}$, signature of the presence of the internal resonance. In that region of the parameter space, a strong coupling between the two modes is at hand with exchange of energy. The dynamics is thus two-dimensional and a single NNM cannot capture such a behaviour.
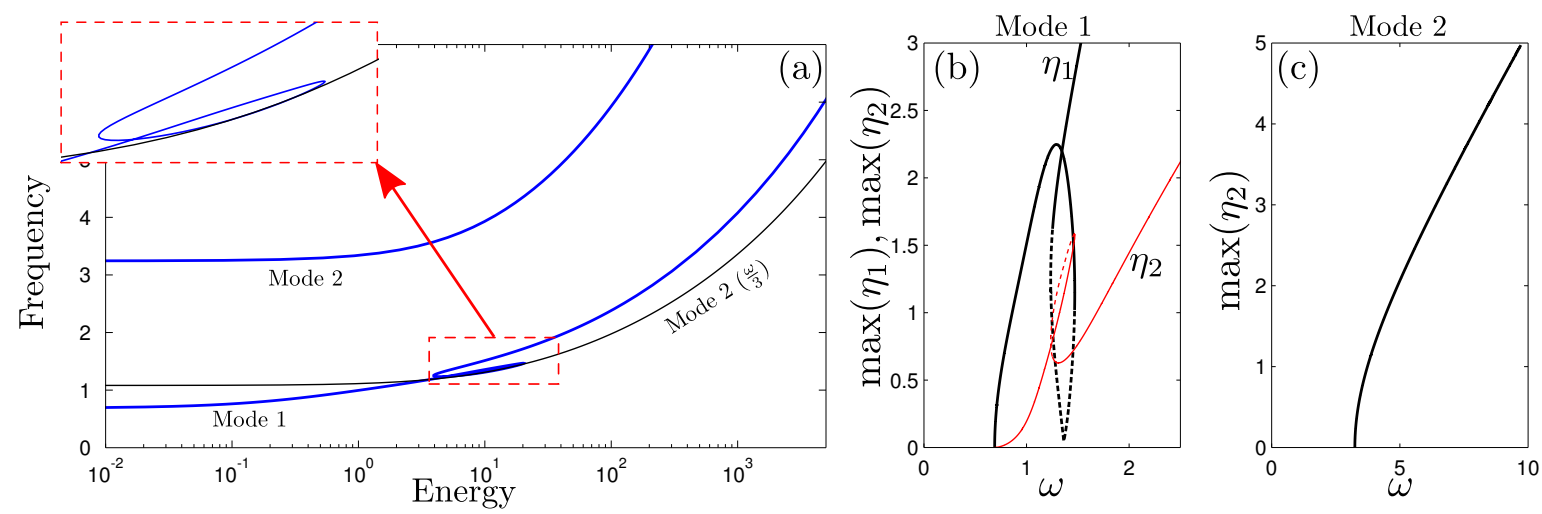

Figure 2: (a): Frequency-Energy plot (FEP) of the system defined by Eqs. (18). Insert: zoom on the 1:3 internal resonance showing the loop of the backbone curve and the emergence of an unstable regime. The 1:3 resonance is assessed by plotting the second mode with the frequency divided by three (thin line). Unstable regions not reported on the FEP. (b-c): backbone curves of the system, maximum amplitude of the master coordinate ( $\eta_{1}$ for mode 1 in (b), $\eta_{2}$ for mode 2 in (c)), versus angular frequency. In (b), $\eta_{2}$ is also represented (thin line) to highlight the internal resonance. Stability reported (dotted lines for unstable solutions).

As a consequence of the internal resonance, the invariant manifold shows also a folding in phase space, as illustrated in Fig. 3 . A section of the manifold at $\dot{\eta}_{1}=0$ shows that the maximum amplitude of the master coordinate $\eta_{1}$ for which a functional relationship of the form $\eta_{2}=f\left(\eta_{1}\right)$ (or in polar form $\eta_{2}=P_{2}(a, \phi)$ as expressed in Eq. (3)) is only possible as long as $\eta_{1} \leq 2.251$. Beyond this value, the center manifold method ceases to provide an operational method for computing the NNM. Consequently, this value will be taken as a limit in amplitude for testing our algorithm, which is consistent with the fact that a 1:3 internal resonance appears so that single-NNM solutions do not exist anymore. It is worth mentioning that all the results shown by Pesheck et al. in [50] are below this amplitude limit. Consequently the 1:3 internal resonance was not highlighted, neither was the fact that this folding point appears as an intrinsic limitation of the center manifold technique. In theory, the invariant manifold technique can be extended to handle internal resonance by considering a higher dimensional mode, with all the master coordinates that are implied in the internal resonance. Here for example a four-dimensional manifold would be needed to handle the 1:3 resonance. However the theory has been derived 
locally for small perturbations around the origin, so that its ability to unfold the manifold when internal resonance involves nonlinear frequencies, is not properly stated and should be examined with care. Secondly, it has been underlined for example in [53] that the multi-mode invariant manifold is more difficult to handle numerically. For these reasons this contribution is limited to single NNM motion.
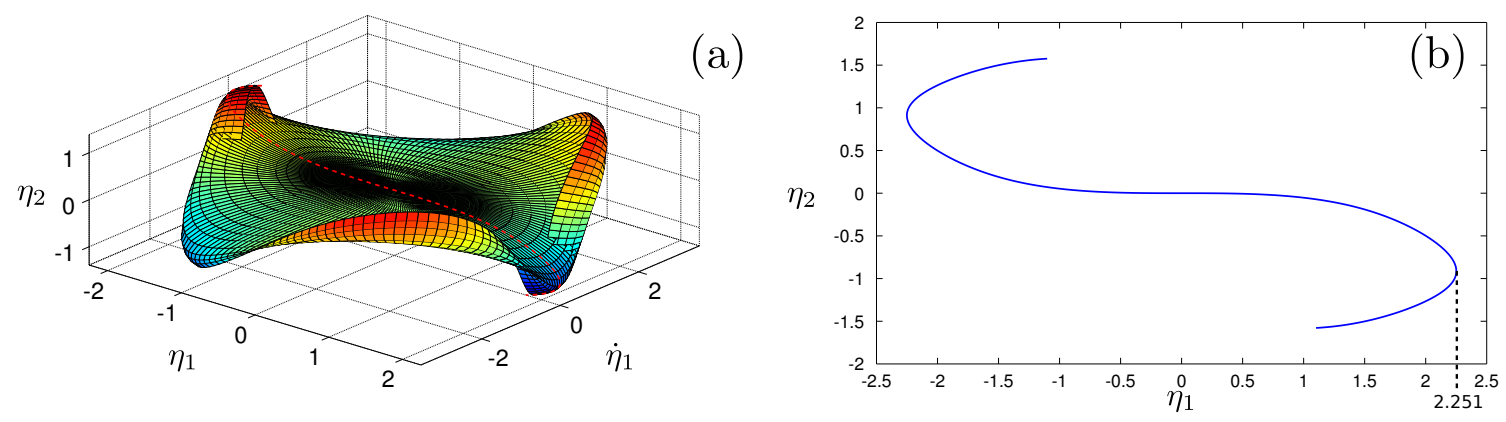

Figure 3: (a) First NNM (invariant manifold) in phase space $\left(\eta_{1}, \dot{\eta}_{1}, \eta_{2}\right)$, just after the folding, showing how the manifold intersects itself. (b): cross-section of the manifold at $\dot{\eta}_{1}=0$ (or equivalently, in polar coordinates, at $\phi=0)$.

Our numerical computation algorithm is tested on the first NNM. As a consequence of the geometry of the manifold and the presence of the folding, the computation is more and more difficult as the value $A_{\max }$ retained for defining the computational domain, is close to 2.25. The first initial condition chosen to compute the NNM is selected as the third-order asymptotic solution, as defined in [13]. For values of $A_{\max }$ in the interval [0.1, 1.8], the convergence to the exact solution is very quick, only a few iterations are needed. As $A_{\max }$ is increased in the range $[1.8,2.2]$, the number of loops in the algorithm increases substantially so that the computation becomes more and more time-consuming. A result is shown in Fig. 4, which has been obtained for $A_{\max }=2.19$, and a computational domain defined by a grid of $N_{a}=$ $80 \times N_{\phi}=1000$ points. With these parameters the computation reaches the (user-defined) minimum tolerance $\left(10^{-10}\right)$ after only a few iterations. Fig. 4 shows the invariant manifold for the displacement $\eta_{2}=P(a, \phi)$ for qualitative comparison with the results shown by Pesheck, as well as a cross-section for quantitative comparison, where the asymptotic (third-order) solution, as well as the result by Pesheck, have been included. One can observe that the asymptotic solution gives quantitative errors that are corrected by using numerical calculation. For our transport method, some difficulties are encountered to converge to the exact solution as $A_{\max }$ approaches the limit value where the vertical tangency is given, whereas the results obtained by Pesheck are coincident with the exact solution up to $a=2.2$.

The difficulties encountered for reaching out convergence of the numerical method may be better understood by looking at the flow of the master mode. As already pointed out in section 2.2 (see Fig. 1), at the boundary $a=A_{\text {max }}$, the flow can be directed inward or outward the computational domain. Another point of view consists in plotting the family of periodic orbits (obtained by numerical continuation), in the same polar coordinates $(a, \phi)$ used for computation, see Fig. 5. One can observe that the trajectories of the system defines naturally the continuation mesh, obtained by concatenating the whole family of periodic orbits. On the other hand, the transport method is defined on a rectangular mesh in $(a, \phi)$, unable to follow the large variations of the orbits outside the domain (for $\phi$-values for which an outward flow was obtained). This problem of mesh definition is common to all numerical methods used to compute NNMs on a 

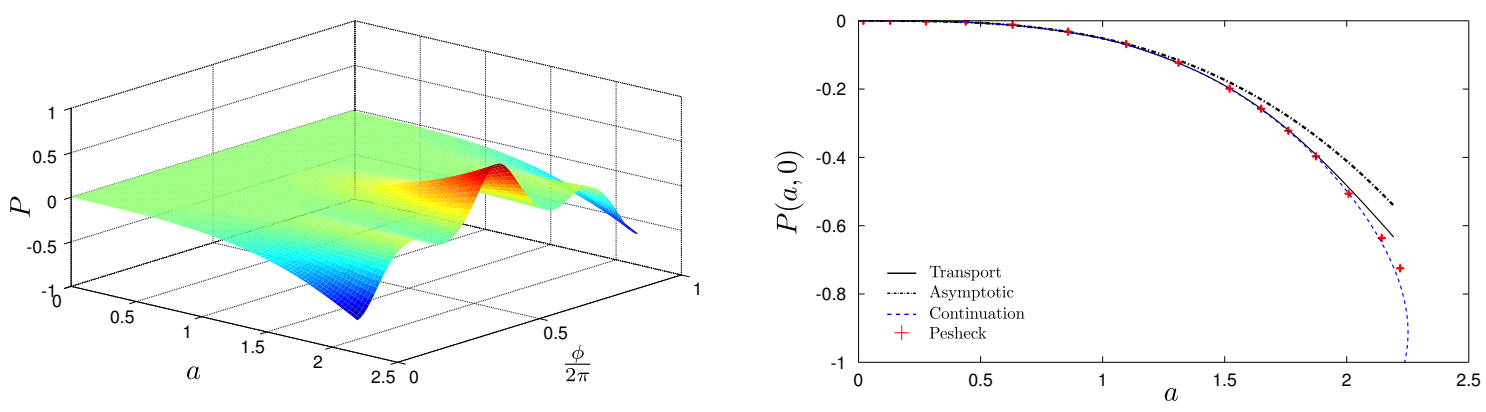

Figure 4: (a): First NNM manifold in polar coordinate, representation of $\eta_{2}=P(a, \phi)$, for a computation realized with $A_{\max }=2.19$. (b): cross-section of $P(a, \phi)$ for $\phi=0$, with comparison to the exact solution obtained by continuation of periodic orbits (dashed blue line), solution obtained by the transport method (plain black line), asymptotic solution (dashed-dotted black line) and solution obtained by Pesheck et al. (crosses).

given grid that do not fit perfectly the domain defined by the trajectories, which cannot be known a priori. However this limitation seems to be particularly effective for the transport method as the folding point is approached, because the algorithm is more sensitive to the in-flow that has to be given at the boundary $a=A_{\max }$. The assumption of extrapolation used at those points becomes here problematic as the folding point is approached.

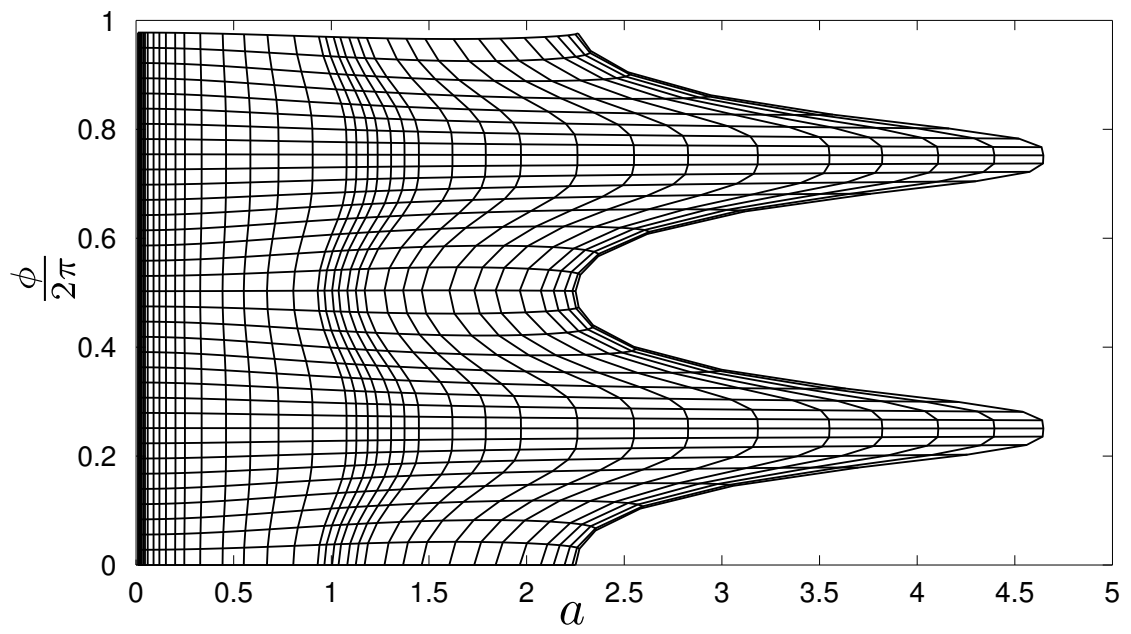

Figure 5: Periodic orbits of the system, obtained by numerical continuation, and represented in polar coordinates $(a, \phi)$.

Computation of the second NNM has also been realized with the transport method. Contrary to the first mode, this NNM do not present any topological difficulty. Consequently, the computations gives very satisfactory results up to very large amplitudes. Calculations up to $A_{\max }=4$ have been realized, for which a quick convergence in a few iterations has been observed. Comparison with the reference solution obtained by continuation, or with the results obtained by Pesheck et al. [50] (limited to an amplitude of 3), shows a perfect coincidence.

This first example has shown that the transport method gives satisfactory results. Difficulties are met when topological problems such as foldings due to internal resonance are encountered. 
Two main problems have been identified. The first one is due to the folding of the manifold, which violates the first assumption of the center manifold technique (a functional relationship between the slave and the master coordinates). Hence this limitation is common to all the numerical techniques used to compute the NNMs of a system that rely on the center manifold method. The second difficulty is linked to the grid definition used in the numerical method. We have underlined that the best grid definition should follow the trajectories of the system that are a priori unknown. This is realized by numerical continuation, but appears to be more difficult to implement, in particular for the transport method. The problem of the boundary condition where in-flow is present is then a consequence of this grid definition.

The main objective of a numerical computation of NNMs in this work, is to derive efficient reduced-order models (ROMs) based on invariant manifolds and center manifold reduction. This is the topic of the next section, where we will underline that a perfect computation of the NNM up to the folding point is not mandatory for obtaining accurate ROMS that are able to predict the backbone curve with fewer degrees of freedom, up to the internal resonance.

\section{Reduced-order models on two-dof examples}

The aim of this section is to derive a single oscillator equation (the so-called reduced-order model, ROM), that would account for the dynamics of the complete system, so that the original multi-dof system can be easily replaced by a ROM with a single equation exhibiting the same dynamical behaviour. As conservative systems are considered, the accuracy of the ROM is judged on its ability to recover properly the backbone curve of the complete system. Whatever the numerical method used for computing the invariant manifold (transport method, continuation, ...), the framework of center manifold theorem can be used. The functional relationship between slave and master coordinates has however to be explicited carefully, as from a numerical result, one would like to recover analytic expressions accounting for the nonlinear restoring force onto the manifold. The first subsection explains how this step can be managed with fitting procedures, then numerical results are given.

\subsection{Methodology}

The center manifold theorem provides the key for deriving the ROMs: once the NNM manifold computation has been realized, one has just to replace all the slave coordinates $\left(\eta_{k}, \dot{\eta}_{k}\right)$ by their respective computed functional relationship with the master coordinate, i.e., $\left(P_{k}(a, \phi), Q_{k}(a, \phi)\right)$. As we assumed that the nonlinear stiffness terms $f_{k}(\boldsymbol{\eta})$ only depends on the displacement (note that this assumption is only for simplicity and is not restrictive), the ROM simply reads:

$$
\ddot{\eta_{p}}+\omega_{p}^{2} \eta_{p}=f_{p}\left(P_{1}(a, \phi), P_{2}(a, \phi), \ldots, \eta_{p}=a \cos \phi, \ldots, P_{N}(a, \phi)\right) .
$$

In order to obtain the reduced dynamics as an oscillator equation, the goal is to obtain a right-hand side term depending only on $\eta_{p}$ and $\dot{\eta}_{p}$. We note $f_{p}(a, \phi)$ the right hand side of (20) and $\hat{\hat{f}}_{p}\left(\eta_{p}, \dot{\eta}_{p}\right)$ the expression we are looking for. We have obtained numerically $f_{p}(a, \phi)$ on a regular grid in the polar coordinates and we need to determine $\hat{\hat{f}}_{p}\left(\eta_{p}, \dot{\eta}_{p}\right)$ on a regular grid in the cartesian coordinates. Since a regular grid in the $(a, \phi)$-plane corresponds to a non uniform grid in the $\left(\eta_{p}, \dot{\eta}_{p}\right)$ plane, with a larger density of points near the equilibrium point, we are lead to proceed in two steps. The first step consists in approximating $f_{p}(a, \phi)$ with:

$$
\hat{f_{p}}(a, \phi)=\sum_{n=0}^{K_{1}} \sum_{k=0}^{K_{2}} g_{n k} a^{k} e^{2 i \pi n \phi},
$$


This is achieved by performing for each $a$-value a Fourier expansion along the $\phi$ coordinate and then by fitting for each $n$-value the coefficients $g_{n k}$ in the $a$-direction. Thanks to the determination of $\hat{f}_{p}(a, \phi)$ the right hand side can be easily evaluated for any $(a, \phi)$ value. The second step consists in evaluating the right hand side on a regular grid in the $\left(\eta_{p}, \dot{\eta}_{p}\right)$ plane. This is realized by first selecting a cartesian domain in the form $\left(\eta_{p}, \dot{\eta}_{p}\right) \in[0, R] \times\left[0, R \omega_{p}\right]$ (in order to have a single parameter $R$ for the size of this grid). Then, thanks to the expression (21), the obtained surface can be fitted with polynomials in the $\eta_{p}$ and $\dot{\eta}_{p}$-directions, leading to:

$$
\hat{\hat{f}}_{p}\left(\eta_{p}, \dot{\eta}_{p}\right)=\sum_{m=0}^{M} \sum_{l=0}^{M-m} s_{m l} \dot{\eta}_{p}^{l} \eta_{p}^{m}
$$

These two steps are performed using Matlab routines. Finally the ROM is expressed as:

$$
\ddot{\eta}_{p}+\omega_{p}^{2} \eta_{p}=\hat{\hat{f}}_{p}\left(\eta_{p}, \dot{\eta}_{p}\right)
$$

and can be used for deriving dynamical informations from a model containing a single oscillatorequation.

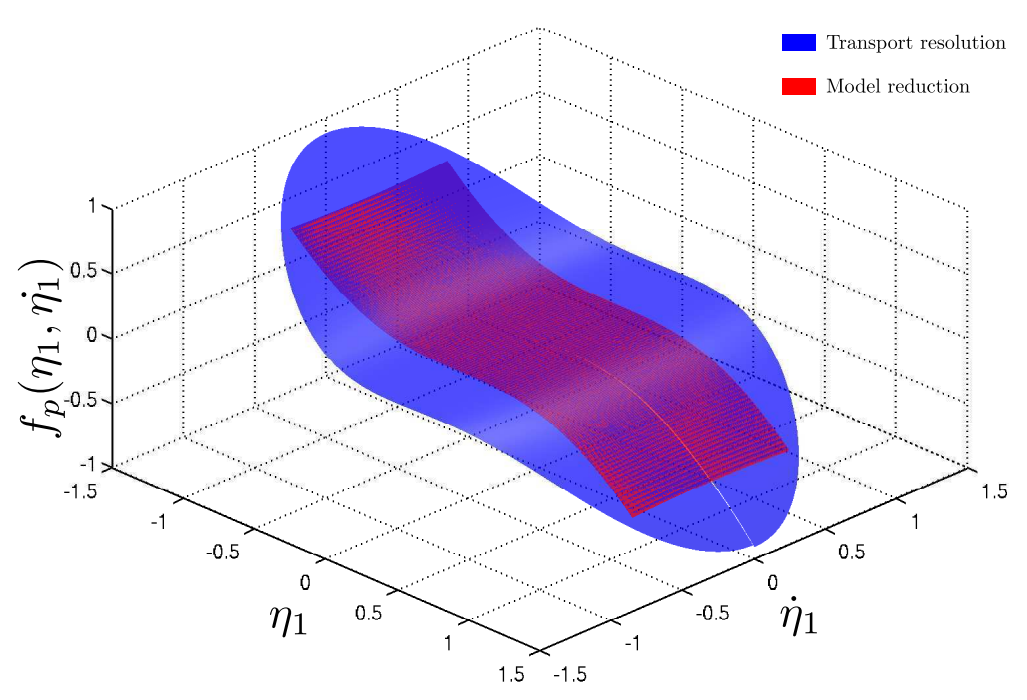

Figure 6: Reduced-order model construction procedure by fitting to the nonlinear terms computed by the numerical algorithm (blue) a polynomial expansion (red, $\left.\hat{\hat{f}}_{p}\left(\eta_{p}, \dot{\eta}_{p}\right)\right)$ of a given degree defined on a given cartesian grid $\left(\eta_{p}, \dot{\eta}_{p}\right) \in[0, R] \times\left[0, R \omega_{p}\right]$. Example of section 2.6, first NNM $(p=1)$ and $R=1.2$.

An illustration of the procedure used to build the ROM is given in Fig. 6, for the example of the previous section 2.6, and the first NNM. Many parameters come into play in the proposed methodology, however the accuracy of the final ROM appears to be particularly sensitive to:

- The choice of the polynomial order $M$ used for expressing $\hat{\hat{f}}_{p}\left(\eta_{p}, \dot{\eta}_{p}\right)$.

- The choice of the maximal value $R$ where the invariant manifold is fitted (or equivalently, to the cartesian grid used to define and fit $\left.\hat{\hat{f}}_{p}\left(\eta_{p}, \dot{\eta}_{p}\right)\right)$. 

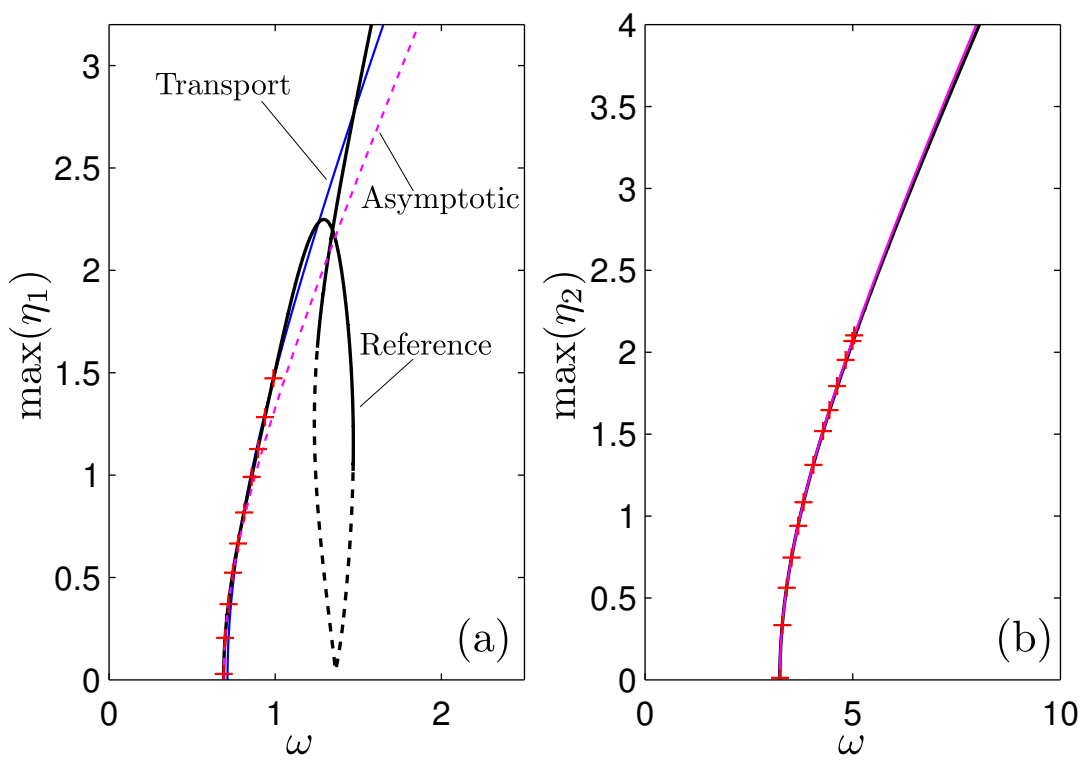

Figure 7: Backbone curves for the first example, (a): first mode, (b): second mode. Comparison between the reference solution (continuation, black line), the prediction given by the ROM computed from the transport method with order 3 (Transport, thin blue line), the asymptotic solution (magenta dashed line) and the solution computed by Pesheck (red crosses).

These points will be discussed on the results obtained on different examples. We have excluded from the discussion the values $K_{1}$ and $K_{2}$ used in the Fourier expansion for fitting the manifold expressed in polar coordinates, as this step appears straightforward. In the remainder, the values $K_{1}=6$ and $K_{2}=5$ have been selected.

\subsection{ROMs of the first example}

We first report the results obtained for the two-dofs system used in section 2.6. The accuracy of the computed ROMs will be assessed by comparing the backbone curve predicted, with the one obtained for the complete system and shown in Fig. 2. Backbone curves are computed by continuation, either on the full system, Eqs. (18), or on the reduced system composed of a single oscillator equation, Eq. (23). Explicit expressions of the oscillator-equations for the different models are given in Appendix B.

Fig. 7 shows the results obtained for the two NNMs. As already underlined, the case of the second mode do not present difficulty. For that mode, even the third-order asymptotic NNMbased ROM gives a very accurate prediction for very large amplitudes. However, as already underlined by Pesheck in that case, differences are observed in the geometry of the exact manifold as compared to the asymptotic one. From amplitudes of the order of 2, discrepancies are noticeable. However they do not give rise to an incorrect prediction of the nonlinear oscillation frequency by the ROM, as revealed by the backbone curve, but important differences are obtained on the amplitude of simulated responses, between the reference and the ROM solution. The same observation has been made with our numerical computation, but for larger amplitudes than those proposed in [50].

The case of the first NNM is much more interesting due to the presence of the internal resonance. Fig. 7(a) shows that the asymptotic NNM-based ROM begins to depart from the exact solution for an amplitude of 1.5 , and then does not see the internal resonance. The prediction 

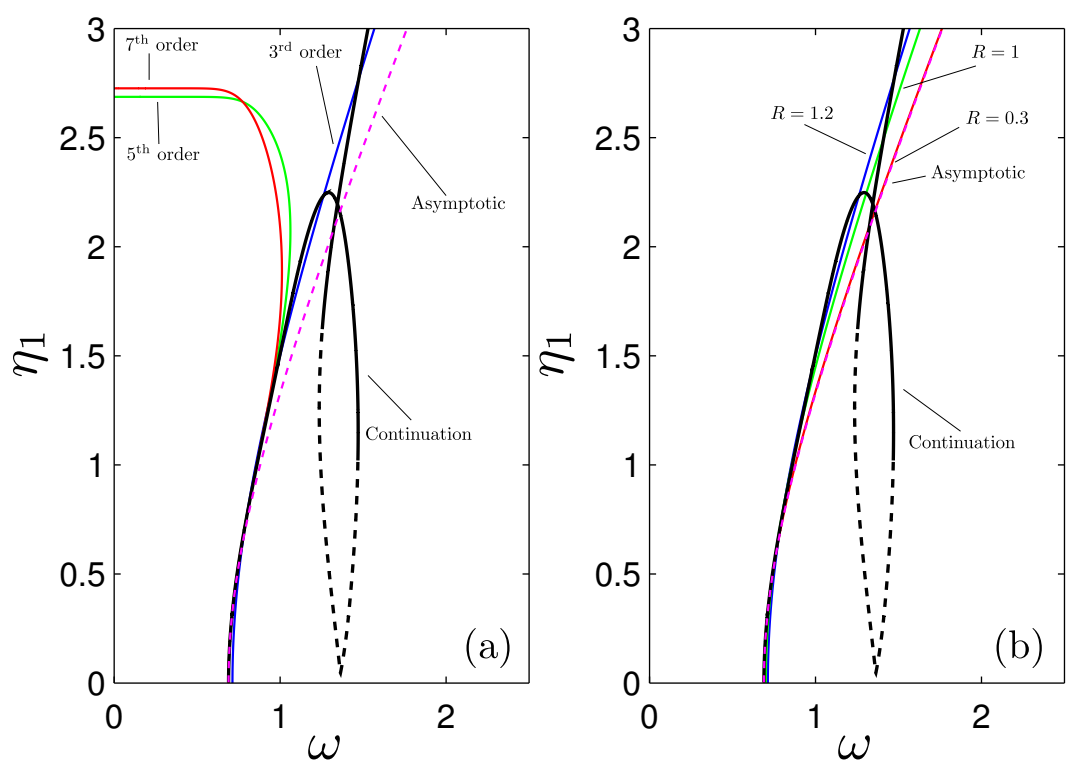

Figure 8: Influence of the parameters used to build the ROM on the backbone curves. (a): polynomial order $M$ for reduction, and comparison between : exact solution (continuation, black line), third-order asymptotic ROM (magenta dashed line), numerical ROM with transport method, and $M=3$ ( $^{\text {rd }}$ order, blue solid line), $M=5$ ( ${ }^{\text {th }}$ order, green solid line), and $M=7$ ( $7^{\text {th }}$ order, red solid line). (b): size of the fitting domain: $R=0.3$ (coincident with the asymptotic solution), $R=1$ and 1.2 .

given in [50] also stops at an amplitude of 1.5 and misses the most important part of the backbone curve. A ROM obtained by the transport method and the proposed methodology, shows that a very good agreement is found up to an amplitude of 2.1, i.e. very close to the folding point. This ROM has been computed by selecting a polynomial order of 3 (which means that the monoms of the form $\dot{\eta}_{p}^{l} \eta_{p}^{m}$ in Eq. (22) are such that $m+l$ do not exceed 3), and the cartesian grid used to fit the manifold was $\left(\eta_{1}, \dot{\eta}_{1}\right) \in[0,1.2] \times\left[0,1.2 \times \omega_{1}\right]$

In fact for amplitudes larger than 2.1, the 1:3 internal resonance dominates the dynamics, so that the ROM, by definition, is not able to catch the backbone curve. Hence the obtained result is, in that sense, the best that could be obtained with a single-dof ROM, as we are accurate up to the folding point where the 1:3 internal resonance appears.

The influence of the parameters used to build the ROM is illustrated in Fig. 8. The polynomial order reduction $M$ is first investigated, for a given cartesian domain $\left(\eta_{1}, \dot{\eta}_{1}\right) \in\left[\begin{array}{ll}0 & 1.2] \times\end{array}\right.$ [0 $1.2 \times \omega_{1}$ ] used to fit the surface. With $M=5$ and $M=7$, the backbone tends to depart from the exact solution for a smaller amplitude than for $M=3$, and thus do not improve the ROM. This behaviour highlights the fact that the surface shows essentially a cubic dependence which is not precisely predicted by the asymptotic solution, but not improved by increasing the order of the polynomial fit. Secondly, for $M=5$ and $M=7$, one observes a sudden change in behaviour when approaching the folding point. This is a reflection of the fact that the polynomial fit has a validity range limited to the domain where the fit has been computed, so that when the topological accident due to the folding appears, for amplitudes larger than those permitted by the fitting domain, the predicted solution diverges. This behaviour appears finally consistent with the fact that something important has happened (an internal resonance), and the proposed $\mathrm{ROM}$ is not able to catch it. 


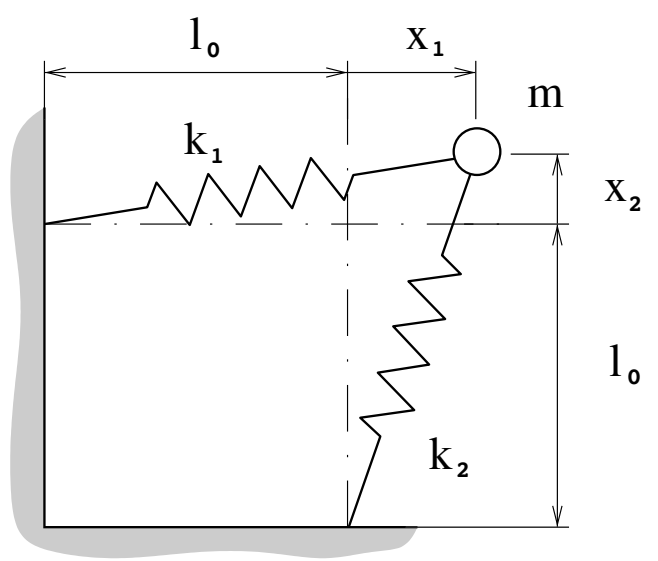

Figure 9: Schematics of the system containing quadratic and cubic nonlinearities

The influence of the fit domain size is more obvious and easy to interpret. The ROM is fitted on rectangular domains $\left(\eta_{p}, \dot{\eta}_{p}\right) \in[0, R] \times\left[0, R \omega_{p}\right]$, with $R=1.2,1$ and 0.3 , with $M=3$. As $R$ decreases, the high amplitude behaviour of the ROM is less taken into account, and the backbone tends to depart earlier from the reference curve, and come closer to the asymptotic solution. For $R$-values larger than 1.2, the ROM begins to deteriorate, so that the results have been shown here with a maximal value of $R=1.2$, which has been found to give the best ROM. However, one must keep in mind that due to the change from polar to cartesian coordinates to build the ROM, the corner values of the cartesian grid are very close to an orbit that have its origin (for $\phi=0$ ) for a larger value of $R$. For example, Fig. 6 illustrates that selecting $R=1.2$ allows fitting an ellipsoidal manifold where the last periodic orbit has an amplitude of 1.5 for $\phi=0$. Values of $R$ larger than 1.2 enforces to fit the manifold in the vicinity of its folding point, which could appear for smaller values in other cut planes than $\phi=0$. This highlights the fact that for building an accurate reduced-order model, there is no need to have a perfect computation of the manifold up to the folding point.

\subsection{A system with quadratic and cubic nonlinearities}

A second example with quadratic and cubic nonlinearity is selected to test our algorithm. The system, composed of a mass connected to two nonlinear springs, is represented in figure 9 . The dynamics of the system is described by :

$$
\left\{\begin{array}{l}
\ddot{X}_{1}+\omega_{1}^{2} X_{1}+\frac{\omega_{1}^{2}}{2}\left(3 X_{1}^{2}+X_{2}^{2}\right)+\omega_{2}^{2} X_{1} X_{2}+\frac{\omega_{1}^{2}+\omega_{2}^{2}}{2} X_{1}\left(X_{1}^{2}+X_{2}^{2}\right)=0, \\
\ddot{X}_{2}+\omega_{2}^{2} X_{2}+\frac{\omega_{2}^{2}}{2}\left(3 X_{2}^{2}+X_{1}^{2}\right)+\omega_{1}^{2} X_{1} X_{2}+\frac{\omega_{1}^{2}+\omega_{2}^{2}}{2} X_{2}\left(X_{1}^{2}+X_{2}^{2}\right)=0,
\end{array}\right.
$$

where $X_{1}=x_{1} / l_{0}$ and $X_{2}=x_{2} / l_{0}$ have been nondimensionalized by the length $l_{0}$ of the springs at rest. The system is fully parameterized by the two eigenfrequencies $\left(\omega_{1}, \omega_{2}\right)$. Depending on their values, the two NNMs can display either hardening or softening behaviour, as shown in [13]. In particular, it is emphasized that for mode 1, the system generally displays a softening behaviour, except in a very narrow region of the parameter space. A thorough comparison of the accuracy of the asymptotic solution as compared to numerical solutions obtained by continuation, is also shown in [57]. This example differs from the first one because of the presence of two fixed points at $\left(X_{1}, X_{2}\right)=(0,0)$ and $(1,1)$, as well as the presence of unstable 

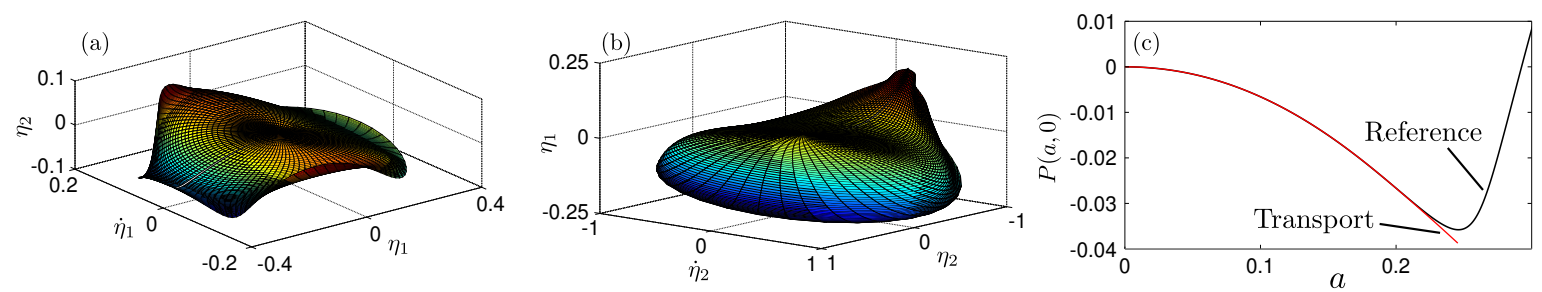

Figure 10: Invariant manifolds (NNMs) of the second example, a two-springs system, computed numerically by continuation of periodic orbits. (a): first mode, (b): second mode, (c): cut-off of the first mode comparing the reference solution (continuation, black line) with the solution computed by the transport method $\left(A_{\max }=0.245\right.$, red line).

fixed points, solutions of:

$$
\left\{\begin{array}{l}
X_{2}=-X_{1}-1 \\
2\left(\omega_{1}^{2}+\omega_{2}^{2}\right) X_{1}^{3}+6 \omega_{1}^{2} X_{1}^{2}+\left(5 \omega_{1}^{2}-\omega_{2}^{2}\right) X_{1}+\omega_{1}^{2}=0 .
\end{array}\right.
$$

Depending on the parameter values, up to three unstable fixed points can be found. When $\omega_{1}=\omega_{2}$ there is a single unstable fixed points at $\left(X_{1}, X_{2}\right)=(-0.5,-0.5)$. Hence this system can be viewed as prototypical of bistable systems, where two stable points are separated by an unstable one. In that case, the stable and unstable manifolds of the unstable fixed points are important in order to understand the dynamics in the vicinity of the fixed points (distribution and stability of the periodic orbits), but this topic is beyond the scope of this paper which is restricted to NNMs.

The parameter values have been selected as: $\omega_{1}=\sqrt{0.5}$ and $\omega_{2}=\sqrt{6}$. The two modes present softening behaviour. Figure 10 shows the manifolds computed by continuation. Their topology are complex, as the first NNM presents inflexion points (see the cut of the manifold) and the second one folds for small amplitude values. The cut on the first mode, Fig. 10(c), shows that our computation deviates from the reference solution where the tangent is horizontal. In fact, as one can see on the three-dimensional view of the manifold, Fig. 10(a), for this radius, the manifold starts to fold at $\phi= \pm \frac{\pi}{2}$.

The backbone curves for the two modes, computed by continuation, are displayed in figure 11. The first mode presents softening-type nonlinearity, with a change in curvature for an amplitude of 0.26 . Then the periodic orbits are unstable for $\max \left(\eta_{1}\right)=0.38$, just before the folding point. It is interesting to note here that the folding is not due to an internal resonance, as in the previous example. Here the folding appears to be a consequence of the presence of unstable fixed points. The second mode shows also softening behaviour, and loses stability for an amplitude of 0.3 .

The methodology described in section 3.1 is applied to this example. Here the optimal ROMs has been obtained for polynomials of order 5. The cartesian grid was defined with $R=0.245$ for the first mode, and $R=0.28$ for the second one. The backbones obtained with these ROMs are shown in Fig. 11, where they are compared to the reference and asymptotic (third-order) solutions. For the first mode, the asymptotic approximation gives a very good prediction until amplitudes of the order of 0.25 , i.e. it misses the change in curvature already underlined. On the other hand, the numerically-built ROM catches very well the change in the backbone curve, but shows then a divergent behaviour, as already observed in the first example.

The backbone of the second NNM is smoother and the asymptotic computation gives satisfactory results. However, the transport computation increases the accuracy of the asymptotic 

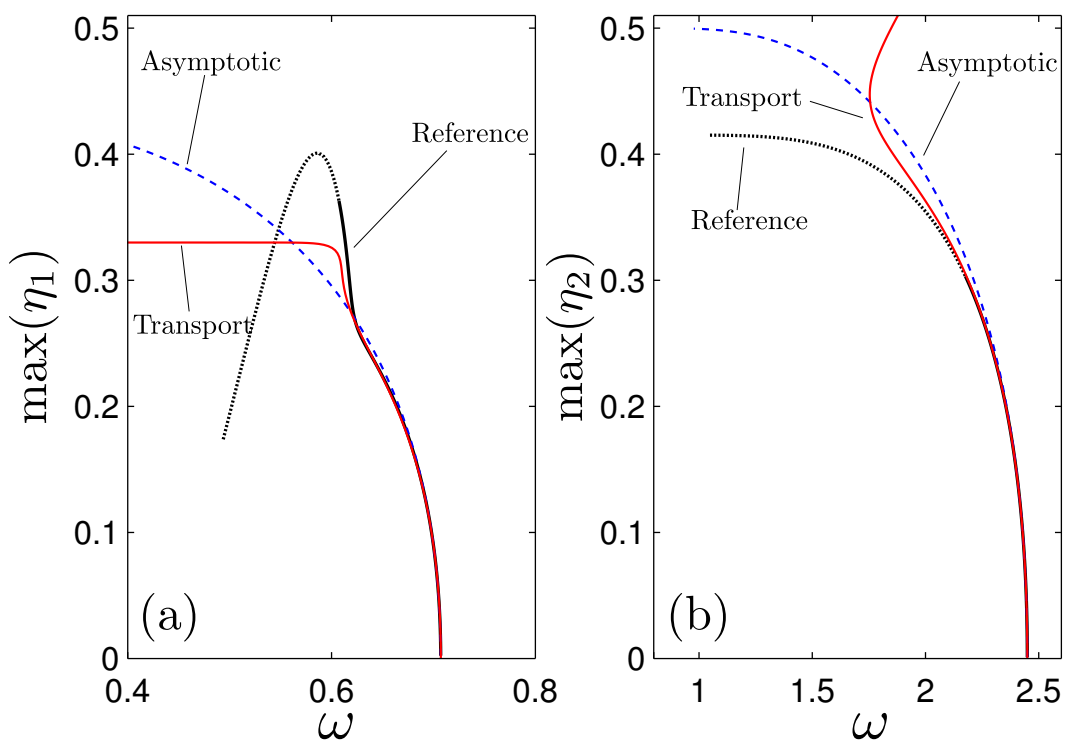

Figure 11: Backbones curves for the second example, (a): first mode, (b): second mode. Reference solution in black, computed by continuation, compared to predictions given by asymptotic third-order ROM (blue dashed line) and given by the numerical procedure (transport method, solid red line).

solution, and follows the reference beyond the last stable orbit. However, it is noteworthy that the ROM cannot restitute the loss of stability.

Two other cases have been treated, respectively with $\omega_{1}=\sqrt{1.7}, \omega_{2}=\sqrt{6}$ (second case), and $\omega_{1}=1, \omega_{2}=\sqrt{3}$ (third case); the obtained results are summarized in figure 12, where the first NNM for these two cases have been reported. In each case, the second NNM has not been represented as their behaviour is more simple and completely in the line of the result obtained in Fig. 11(b), i.e. showing an improved prediction as compared to the asymptotic solution, which already gives a very satisfactory result. The first mode for case 2 is interesting as a change from hardening to softening behaviour is observed on the reference solution. The asymptotic third-order ROM reproduces this change but for a very large amplitude value so that from an amplitude of 0.1 the solution is not reliable anymore. On the other hand, the numericallybuilt ROM reproduces well the overall behaviour and departs from the reference solution in the vicinity of the folding point. For the third case, it is also found that the numerically-built ROM is able to reproduce perfectly the backbone curve up to the folding point.

The discussion on these two examples have shown a global methodology to derive accurate ROMs from a numerical computation of invariant manifolds. Discussions on the sensitive parameters used to derive the ROM have been conducted, showing that the domain used to fit the polynomials might be large enough to improve the asymptotic solution, but small enough to prevent to fit the manifold on folding points. In general, a grid substantially smaller than the domain of existence of the functional link between slave and master coordinates, is sufficient. Moreover, it has been shown that polynomials of low orders (5 or less) are generally accurate enough to compute the ROM, and that increasing the order may not improve the results. Some of the computed ROMs give backbones that diverge when approaching foldings. This is consistent with the fact that unimodal solutions do not exist anymore in these areas. 

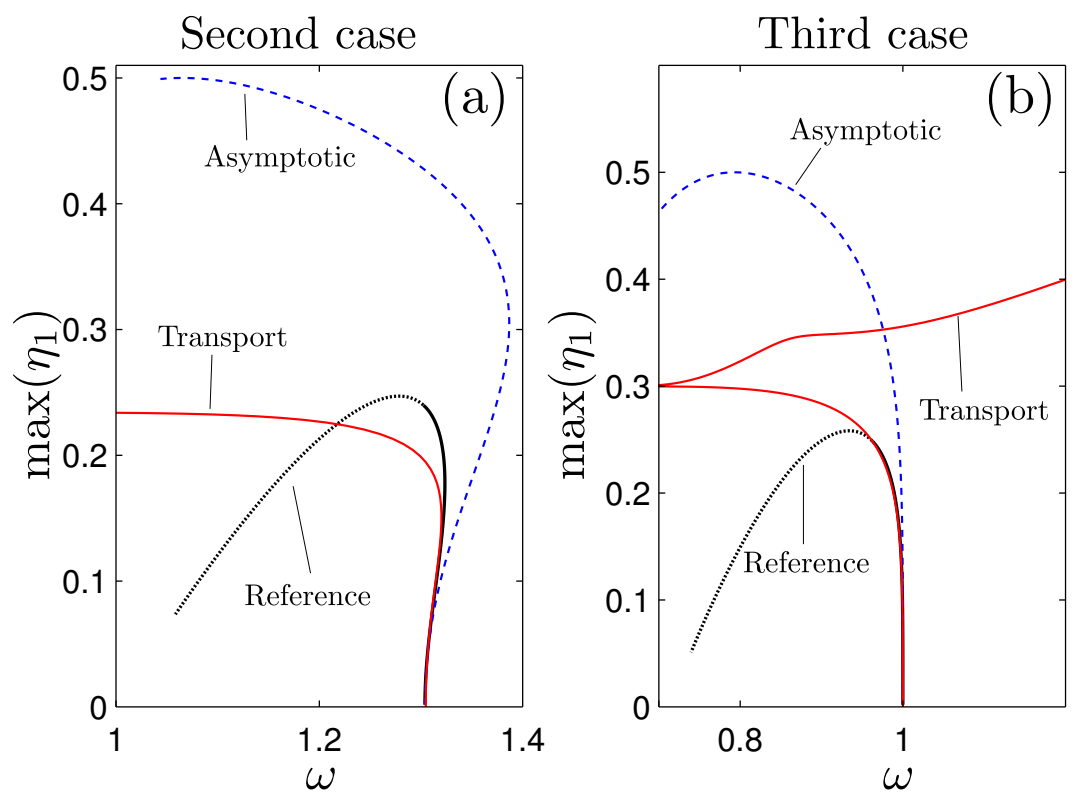

Figure 12: Backbone curves of the second example, (a): $\omega_{1}=\sqrt{1.7}, \omega_{2}=\sqrt{6}$, first NNM; (b): $\omega_{1}=1, \omega_{2}=\sqrt{3}$, first NNM. Comparisons between reference solution (black line, unstable solutions with dotted line), transport method (thin red line) and asymptotic solution (dashed blue line).

We conclude with an example involving a continuous structure.

\section{A continuous problem}

\subsection{Equations of motion}

The last example is a linear Euler-Bernoulli beam, hinged at its two ends, and resting on a nonlinear elastic foundation with quadratic and cubic distributed nonlinearities. In nondimensional form, the undamped transverse vibrations are governed by [14]:

$$
\frac{\partial^{2} w}{\partial t^{2}}+\frac{\partial^{4} w}{\partial x^{4}}+\alpha_{2} w^{2}+\alpha_{3} w^{3}=0 .
$$

$w(x, t)$ is the nondimensional transverse displacement (where the characteristic length used is the thickness $h$ of the beam), $\alpha_{2}$ and $\alpha_{3}$ are two parameters. The boundary conditions read:

$$
w(x, t)=0, \quad \frac{\partial^{2} w(x, t)}{\partial x^{2}}=0 \quad \text { for } \quad x=0,1 .
$$

The linear analysis provides the eigenmodes as well as the eigenfrequencies:

$$
\begin{aligned}
& \Phi_{n}(x)=\sqrt{2} \sin (n \pi x), \\
& \omega_{n}=n^{2} \pi^{2} .
\end{aligned}
$$

Denoting $X_{p}$ the modal co-ordinate associated to the $p^{\text {th }}$ linear mode, the projection yields the following problem, $\forall p=1, \ldots, N$ :

$$
\ddot{X}_{p}+\omega_{p}^{2} X_{p}+\sum_{i, j=1}^{N} \tilde{g}_{i j}^{p} X_{i} X_{j}+\sum_{i, j, k=1}^{N} \tilde{h}_{i j k}^{p} X_{i} X_{j} X_{k}=0
$$


where:

$$
\tilde{g}_{i j}^{p}=\alpha_{2} \int_{0}^{1} \Phi_{i}(x) \Phi_{j}(x) \Phi_{p}(x) \mathrm{d} x, \quad \tilde{h}_{i j k}^{p}=\alpha_{3} \int_{0}^{1} \Phi_{i}(x) \Phi_{j}(x) \Phi_{k}(x) \Phi_{p}(x) \mathrm{d} x .
$$

Eq. (30) is made similar to Eq. (1) by considering the symetries of the nonlinear quadratic and cubic terms (e.g. for $j>i, g_{i j}^{p}=\tilde{g}_{i j}^{p}+\tilde{g}_{j i}^{p}$ and $\left.g_{j i}^{p}=0\right)$. For the simulation, the parameters have been set to $\alpha_{2}=12$ and $\alpha_{3}=0.5$, so that the behaviour will be first of the softening type due to the large positive value of $\alpha_{2}$. Then, for very large amplitudes, the cubic term will dominate and a hardening behaviour will be recovered. These values have been chosen to have a third kind of behaviour, after the hardening system (first example), and the softening example typical of bi-stable systems with loss of stability. This last example will allow to test our method on a continuous system with a change of nonlinear behaviour, from softening to hardening.
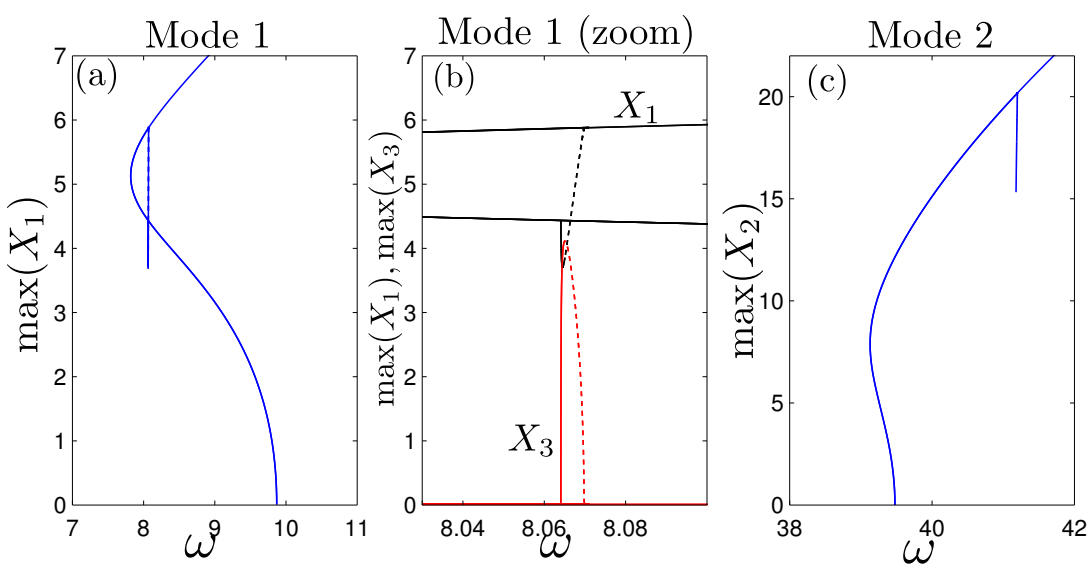

Figure 13: Backbone curves for the first and second mode of the beam, computed by continuation for reference solutions. (a): first mode, computed with a truncation involving four linear modes. (b): zoom on the tongue of 1:3 internal resonance between the first $\left(X_{1}\right.$, black line) and the third modes $\left(X_{3}\right.$, red line). (c): second mode (truncation with five linear modes).

The backbone curves for the first two modes, computed by continuation, are shown in Fig. 13. As announced, a softening behaviour is found for low amplitudes, and then turn back to hardening. Two internal resonances are found to appear. For the first NNM, the tongue of internal resonance involve a 1:3 relationship with the third mode. Figure 13(b) shows a zoom on the portion where the internal resonance is activated, giving rise to a short tongue that is sharp and difficult to detect for the continuation method, with a sudden increase of the third coordinate $X_{3}$. For the main coordinate $X_{1}$, the branch of internal resonance connects the main branch of periodic orbits at the amplitudes $\max \left(X_{1}\right)=4.4$ and 5.8. For the first NNM, the result shown has been computed with four linear modes retained in the truncation, convergence has been checked as the backbone does not vary when adding more modes. For the second mode, a tongue of internal resonance with the fifth mode has been found for very large amplitude of vibrations $\left(\max \left(X_{2}\right)=20.18\right)$. The model retains in that case 5 linear modes in truncation.

\subsection{Reduced-order models for mode 1 and 2}

The reduction procedure presented in the previous section is now applied, for mode 1 to the system composed of the first four linear modes, and for mode 2 to a truncation with 5 modes. 
For the first NNM, the numerical computation of the manifold's geometry with the transport algorithm converges easily for amplitudes up to 4.3. Then the convergence was not obtainable. These observations are coherent with the fact that an internal resonance occurs for an amplitude of 4.4, thus a folding point in the geometry should be present so that the convergence is not possible as the functional relationship does not exist anymore. For the second mode, computations up to an amplitude of 19 have been realized and convergence was easily obtained.
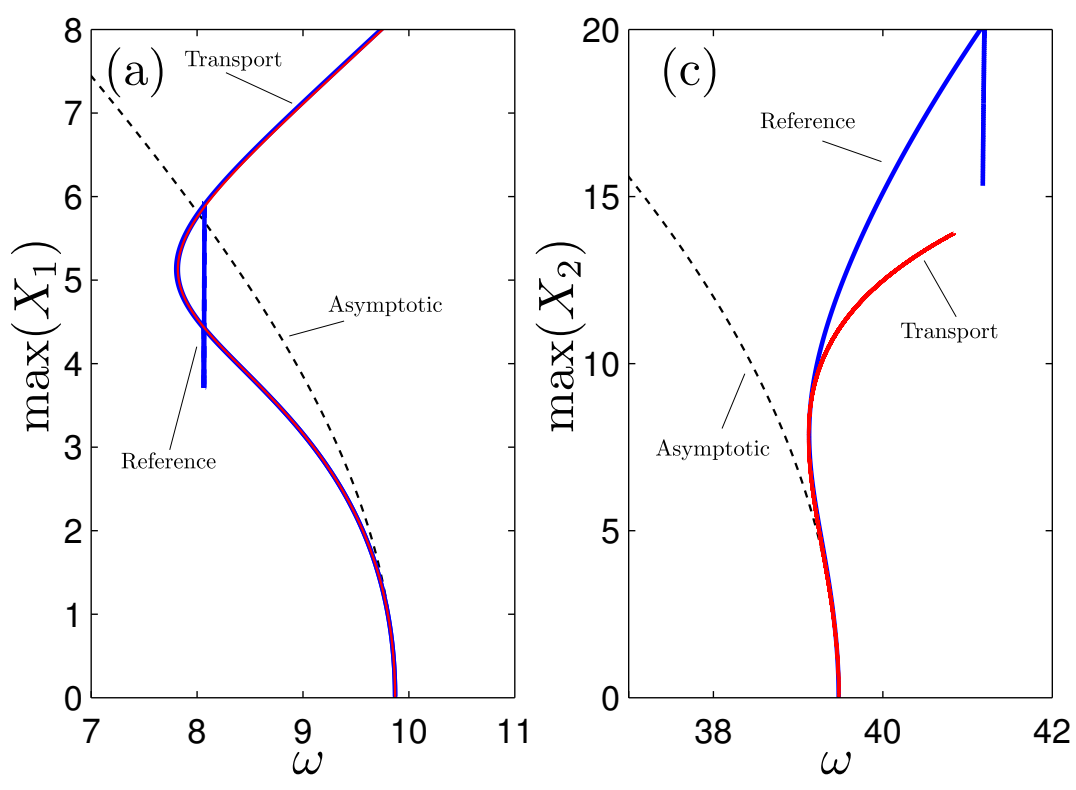

Figure 14: Comparison of backbone curves, reference solution (blue solid line), numerical ROM (Transport solution, red line) and asymptotic ROM (dashed black line). (a): first mode, (b): second mode.

The ROMs are built using the fitting procedure explained in section 3.1. For the first mode, the best ROM was obtained by fitting the manifold on a domain defined by $\left(X_{1}, \dot{X}_{1}\right) \in$ $[0,2.3] \times\left[0,2.3 \omega_{1}\right]$, and with a polynomial expansion of order three. The resulting backbone curve is shown in Fig. 14(a). One can see that a perfect result is obtained, as the ROM is able to recover the complete backbone curve, up to very large amplitudes. Logically, the ROM goes over the internal resonance without detecting it as a single dof ROM is constructed. This excellent result has been found for a rather small domain used for fitting the manifold. Using larger domain does not improve the result, as a divergence was observed when approaching the branch of internal resonance, as observed in the two previous examples. Comparison with the asymptotic third-order NNM shows also that an important gain in accuracy has been realized thanks to the numerical approach.

The results for mode 2 are shown in Fig. 14(b). The best ROM has been constructed by fitting the manifold on a domain defined by $\left(X_{2}, \dot{X}_{2}\right) \in[0,9] \times\left[0,9 \omega_{2}\right]$, and with a polynomial expansion of order three. The geometry of this second NNM has been found more difficult to fit due to the presence of inflexion points in the vicinity of the origin, so that it has not been possible to fit the manifold up to the folding point. Nevertheless, the backbone predicted by the ROM shows a very significant improvement as compared to the asymptotic one: the change from hardening to softening behaviour is detected by the numerical ROM, and a fair accuracy up top a very large amplitude of vibration of 10 is observed (as the characteristic length for nondimensionalizing the equations is the thickness $h$, this means that the ROM is accurate up 
to vibration amplitudes that are ten times the thickness). Moreover the computed ROM is also able to catch the change from softening to hardening nonlinearity, a peculiar feature that was missed by the aymptotic approach.

\section{Conclusion}

A numerical method for computing the NNMs of a mechanical system featuring geometric nonlinearity has been introduced, based on the definition of the NNMs as invariant manifold of phase space, and the center manifold theorem. An interpretation of the PDEs describing the geometry of the manifold in phase space, as a transport equation, enabled to understand interesting peculiarities of this formulation, as well as to propose a numerical scheme involving a periodicity criterion to fulfill. Convergence was obtained via optimization technique and the definition of an adjoint problem. The main objective of the study was to provide a constructive method for defining numerically accurate Reduced-order Models (ROMs) based on the invariant manifold approach, so that a fitting procedure, allowing polynomial expressions of the computed surfaces, has also been proposed.

The study has revealed fundamental limitations for the derivation of ROMs, based on the center manifold idea of a functional relationship between slave and master coordinates. A first limitation has been observed for numerical methods, due to the distorsion of the periodic orbits at large amplitude. We have underlined that the best grid to compute numerically the NNMs should follow the lines of the periodic orbits, so that any numerical method whose computational domain does not fit the orbits will face convergence problems at some points due to some missing informations. Computation of reference solutions for NNMs with continuation methods has also revealed that in general, invariant manifolds display folding points, hence fixing a limit amplitude for the definition of ROMs. Three examples with different characteristics have been studied. The first example is a two-dofs system with cubic (hardening) nonlinearity, showing for the first mode a folding point due to the presence of a 1:3 internal resonance with the second mode. This behaviour appears to be generic, as it has already been observed in many other systems (see e.g. $[6,58,54]$ ), and is also seen in the continuous system used as third example : a linear beam resting on a nonlinear fundation. The second example, a two-dofs system displaying quadratic and cubic nonlinearity, has been selected as it shows a different global behaviour. Due to the presence of two stable positions, in the vicinity of which NNMs exist, and an unstable position inbetween these, this system appears to be characteristic of bi-stable systems. The NNMs are characterized by a softening behaviour, a loss of stability for relatively small amplitude of vibrations, and a folding that is merely due to the presence of the unstable fixed point and the constraints on the periodic orbits. Nonetheless, all the three cases show that the invariant manifolds display folding points that are intrinsic limitations for the construction of single-degree-of-freedom ROMs.

Inside the amplitude limitations due to folding points, the numerical method based on the transport interpretation behaves very well and converge to the exact solution. Together with the fitting procedure developed for building ROMs, we have been able to recover with very good accuracy the backbone curve of the complete system in all the three cases, up to large amplitudes. It is worth mentioning that the asymptotic third-order NNM-based ROMs gives generally acceptable results, and for a very light computation procedure that is much easier to implement and run. Even though the numerical method allows to recover with accuracy the backbone up to (generally) the folding points, the amplitude range between these folding points and the amplitude for which the asymptotic solution depart from the exact one, has been found 
to be somehow small, depending on the selected examples.

The next step is to extend the transport method to damped systems. For that purpose, the general procedure outlined in section 2 could be used a priori without particular changes, as the main features of the method do not fail when damping is added. In particular, the periodicity condition -assessing the continuity of the manifold- still holds, in contrast with methods using periodic orbits (continuation, shooting, ...) which disappear with damping. However, preliminary tests reveal a very difficult convergence, which has been interpreted as a reflection of the fact that when damping is added, in-flow is present along almost all the external boundary at $a=A$. In that context, the simple strategy used in the conservative case, and consisting in extrapolating points where in-flow is present, is not sufficiently robust anymore. Hence for damped problems a better selection of an adapted grid has to be first tackled. For example, a simple idea would be to change the polar coordinates used in Eqs. (2) for $\eta_{p}=a \mathrm{e}^{-\xi_{p} \phi} \cos \phi$, $\xi_{p}=-a \omega_{p} \mathrm{e}^{-\xi_{p} \phi} \sin \phi$, so that the linear damping would be accounted for in the grid definition. However this choice leads to rewrite all the equations for the direct transport and adjoint problem.

\section{Appendix A. Adjoint state and gradient determination}

The aim of this appendix is to determine the adjoint state from the direct problem, and then to derive the functional gradient. The transport problem we deal with is made particularly difficult due to the fact that the velocity and the right hand side are nonlinear functions of the unknowns. This is in contrast with usual presentations of adjoint determinations that can be found from textbooks, see e.g. [56], where only the periodicity constraint is treated. Therefore, to simplify the presentation, we temporarily consider that the velocity and the source term $f_{2}$ are independant of the unknown $P$, are known and we call them $\vec{V}^{0}(a, \phi)$ and $f_{2}^{0}(a, \phi)$. This allows to define a simpler problem:

The simplified minimization problem: Find $P_{0}(a)$ and $Q_{0}(a)$ such that the cost functional (8) is minimum where $P(a, \phi)$ and $Q(a, \phi)$ are the solutions of the transport problem:

Find $P(a, \phi)$ and $Q(a, \phi)$ solutions of:

$$
\begin{aligned}
\vec{V}^{0} \cdot \vec{\nabla} P-Q & =0, \\
\vec{V}^{0} \cdot \vec{\nabla} Q+\omega_{2}^{2} P & =f_{2}^{0},
\end{aligned}
$$

with the initial conditions $P(a, 0)=P_{0}(a)$ and $Q(a, 0)=Q_{0}(a)$ and the boundary conditions $P(A, \phi)=0=Q(A, \phi)$ for all $\phi$ such that $V_{a}^{0}(A, \phi)<0$.

Note that, also in order to simplify the presentation, we have here considered that at the boundary $a=A$ where in-flux occurs, the unknowns vanishes. The reader must keep in mind that all simplifications introduced in this appendix are only given so as to obtain a progressive introduction to the adjoint problem.

\section{Appendix A.1. Linearized and Adjoint problems}

To define the linearized problem we introduce $p(a, \phi)$ and $q(a, \phi)$ such that $P(a, \phi)+p(a, \phi)$ and $Q(a, \phi)+q(a, \phi)$ are solutions of the simplified transport problem (A.1) associated to the initial conditions $P_{0}(a)+p_{0}(a)$ and $Q_{0}(a)+q_{0}(a)$ for any small perturbations $p_{0}(a)$ and $q_{0}(a)$. Introducing the following functions of $(a, \phi): \gamma_{1}=-V_{a}^{0} / V_{\phi}^{0}, \gamma_{2}=1 / V_{\phi}^{0}$ and $\gamma_{3}=-\omega_{2}^{2} / V_{\phi}^{0}$, 
the linearization process of problem (A.1) leads to the linearized transport problem for the perturbations $p$ and $q$ :

The linearized problem: For $p_{0}(a)$ and $q_{0}(a)$ given functions, find $p(a, \phi)$ and $q(a, \phi)$ solutions of:

$$
\partial_{\phi}\left(\begin{array}{c}
p \\
q
\end{array}\right)=\left[\begin{array}{cc}
\gamma_{1} \partial_{a}(.) & \gamma_{2} \\
\gamma_{3} & \gamma_{1} \partial_{a}(.)
\end{array}\right]\left(\begin{array}{c}
p \\
q
\end{array}\right)
$$

with the initial conditions $p(a, 0)=p_{0}(a)$ and $q(a, 0)=q_{0}(a)$, and the boundary conditions $p(A, \phi)=0=q(A, \phi)$ where $V_{a}^{0}(A, \phi)<0$ (or equivalently $\gamma_{1}>0$ since $\left.\gamma_{1}=-V_{a}^{0} / V_{\phi}^{0}\right)$.

We will now define the adjoint of this linearized problem, which is the last step to determine the functional gradient. Let us introduce the problem:

The adjoint problem: For $\tilde{p}_{2 \pi}(a)$ and $\tilde{q}_{2 \pi}(a)$ given functions, find $\tilde{p}(a, \phi)$ and $\tilde{q}(a, \phi)$ solutions of

$$
-\partial_{\phi}\left(\begin{array}{c}
\tilde{p} \\
\tilde{q}
\end{array}\right)=\left[\begin{array}{cc}
-\partial_{a}\left(\gamma_{1}(.)\right) & \gamma_{3} \\
\gamma_{2} & -\partial_{a}\left(\gamma_{1}(.)\right)
\end{array}\right]\left(\begin{array}{l}
\tilde{p} \\
\tilde{q}
\end{array}\right)
$$

with the end conditions $\tilde{p}(a, 2 \pi)=\tilde{p}_{2 \pi}(a)$ and $\tilde{q}(a, 2 \pi)=\tilde{q}_{2 \pi}(a)$ and the boundary conditions $\tilde{p}(A, \phi)=0=\tilde{q}(A, \phi)$ where $\gamma_{1}(A, \phi)<0\left(V_{a}^{0}>0\right)$.

The notation $\partial_{a}\left(\gamma_{1}().\right)$ is introduced where (.) refers either to $\tilde{p}(a, \phi)$ or to $\tilde{q}(a, \phi)$ depending on the case. For instance, $\partial_{a}\left(\gamma_{1}(\tilde{p})\right)=\partial_{a}\left(\gamma_{1}\right) \cdot \tilde{p}+\gamma_{1} \cdot \partial_{a}(\tilde{p})$.

This adjoint problem has to be solved in the backward sense, from $\phi=2 \pi$ to 0 . Therefore contrary to the direct problem (A.2), this adjoint problem is not defined with initial conditions and note also that at $a=A$ the solution is imposed to be zero where the flux of the direct problem goes out. This is the adjoint problem of the linearized problem (A.2) in the sense that the integral:

$$
I=\int_{0}^{A} \int_{0}^{2 \pi} \partial_{\phi}(p \tilde{p}+q \tilde{q}) \mathrm{d} \phi \mathrm{d} a,
$$

is equal to zero. Indeed using the definitions of the direct problem (A.2) and of the adjoint problem (A.3) we find:

$$
I=\int_{0}^{2 \pi}\left[\gamma_{1}(p \tilde{p}+q \tilde{q})\right]_{a=0}^{a=A} \mathrm{~d} \phi
$$

This term is zero, at $a=0$ since $\gamma_{1}=0$ and moreover at $a=A$ : indeed $p$ and $\tilde{p}$ vanish on complementary domains, when $\gamma_{1}>0$ for $p$ and when $\gamma_{1}<0$ for $\tilde{p}$ (the same for $q$ and $\tilde{q}$ ).

Noting that the expression (A.4) implies that:

$$
I=\int_{0}^{A}[p \tilde{p}+q \tilde{q}]_{\phi=0}^{\phi=2 \pi} \mathrm{d} a
$$

a useful relationship is deduced from the previous result $I=0$ :

$$
\int_{0}^{A}\left[p(a, 2 \pi) \tilde{p}_{2 \pi}(a)+q(a, 2 \pi) \tilde{q}_{2 \pi}(a)\right] \mathrm{d} a=\int_{0}^{A}\left[p_{0}(a) \tilde{p}(a, 0)+q_{0}(a) \tilde{q}(a, 0)\right] \mathrm{d} a .
$$

This expression is the key for evaluating numerically the functional gradient. This is done in the next paragraph. 
Appendix A.2. Gradient determination

satisfying:

The gradient $\vec{\nabla} J=\left[\begin{array}{l}d_{P}(a) \\ d_{Q}(a)\end{array}\right]$ is defined as a couple of functions $a \longmapsto d_{P}(a)$ and $a \longmapsto d_{Q}(a)$

$$
J\left(P_{0}+p_{0}, Q_{0}+q_{0}\right) \simeq J\left(P_{0}, Q_{0}\right)+\int_{0}^{A} d_{P}(a) p_{0} \mathrm{~d} a+\int_{0}^{A} d_{Q}(a) q_{0} \mathrm{~d} a+\mathfrak{r}_{1}
$$

for any small perturbations $p_{0}(a)$ and $q_{0}(a)$ of the initial conditions $P_{0}(a)$ and $Q_{0}(a)$, and where $\mathfrak{r}_{1}$ is a remainder which is at least of order two with respect to the perturbations. Secondly, starting from the functional $J\left(P_{0}, Q_{0}\right)$, as first defined in Eq. (8), we get after a linearization at the first order another expression for $\nabla J$ :

$$
\begin{aligned}
J\left(P_{0}+p_{0}, Q_{0}+q_{0}\right) \simeq & J\left(P_{0}, Q_{0}\right)+\int_{0}^{A}\left[P(a, 2 \pi)-P_{0}(a)\right]\left[p(a, 2 \pi)-p_{0}(a)\right] \mathrm{d} a \\
& +\int_{0}^{A}\left[Q(a, 2 \pi)-Q_{0}(a)\right]\left[q(a, 2 \pi)-q_{0}(a)\right] \mathrm{d} a+\mathfrak{r}_{2},
\end{aligned}
$$

with $\mathfrak{r}_{2}$ a second-order remainder. To link the two expressions of the gradient, we choose the following end conditions for the adjoint problem:

$$
\begin{aligned}
& \tilde{p}_{2 \pi}(a)=P(a, 2 \pi)-P_{0}(a) \\
& \tilde{q}_{2 \pi}(a)=Q(a, 2 \pi)-Q_{0}(a) .
\end{aligned}
$$

Then incorporating (A.10) in relation (A.9), using the relationship (A.7) and comparing to definition (A.8) leads to the expression of the functional gradient:

$$
\vec{\nabla} J\left(P_{0}, Q_{0}\right)=\left[\begin{array}{c}
\tilde{p}(a, 0)-\tilde{p}_{2 \pi}(a) \\
\tilde{q}(a, 0)-\tilde{q}_{2 \pi}(a)
\end{array}\right] .
$$

Therefore the numerical evaluatuation of the functional gradient just requires to solve the adjoint problem from $\phi=2 \pi$ to 0 . This is the main advantage of using the adjoint state as it gives a particularly useful way for simply estimating the gradient in an optimization problem. This ends the full proof for deriving the adjoint problem and the functional gradient, in the simplified problem where the flow is frozen and boundary conditions with incoming flow set to zero. In practice, to get a better convergence in the original problem, it has been found much more efficient to assume regularity of the manifold at $a=A$ where in-flow occurs, so that this choice has been retained and incorporated in the main text. Finally the complete proof for establishing the adjoint state from the original problem is very similar to the one shown here for the simplified problem.

\section{Appendix B. ROMS for the first example}

The different reduced-order models used in section 3.2 are here given explicitely.

- Asymptotic NNM for the first NNM:

$$
\ddot{\eta}_{1}+\omega_{1}^{2} \eta_{1}+0.405 \eta_{1}^{3}=0
$$


- ROM for figure 7(a), first NNM, computed with $\mathrm{M}=3$ and $R=1.2$ :

$$
\begin{aligned}
\ddot{\eta}_{1}+\omega_{1}^{2} \eta_{1} & +0.29698 \eta_{1}^{3}+0.00041563 \eta_{1}^{2}+0.0015994 \eta_{1} \dot{\eta}_{1}^{2} \\
& +0.033312 \eta_{1}+0.00019508 \dot{\eta}_{1}^{2}=0
\end{aligned}
$$

- Asymptotic NNM for the second NNM:

$$
\ddot{\eta}_{2}+\omega_{2}^{2} \eta_{2}+4.58 \eta_{2}^{3}=0
$$

- ROM for figure 7(b), second NNM, computed with $\mathrm{M}=3$ and $R=1.2$ :

$$
\ddot{\eta}_{2}+\omega_{2}^{2} \eta_{2}+4.6373 \eta_{2}^{3}+0.00080388 \eta_{2}^{2}+0.0005063 \eta_{2} \dot{\eta}_{2}^{2}-0.02931 \eta_{2}=0
$$

[1] R. M. Rosenberg. The normal modes of nonlinear n-degree-of-freedom systems. Journal of Applied Mechanics, 29:7-14, 1962.

[2] R. M. Rosenberg. On non-linear vibrations of systems with many degrees of freedom. Advances in Applied Mechanics, 9:155-242, 1966.

[3] N. Boivin, C. Pierre, and S. Shaw. Non-linear normal modes, invariance, and modal dynamics approximations of non-linear systems. Nonlinear Dynamics, 8:315-346, 1995.

[4] C. Gibert. Fitting measured frequency response using non-linear normal modes. Mechanical Systems and Signal Processing, 17(1):211-218, 2003.

[5] D. Jiang. Nonlinear modal analysis based on invariant manifolds. Application to rotating blade systems. PhD thesis, University of Michigan, 2004.

[6] G. Kerschen, M. Peeters, J.C. Golinval, and A.F. Vakakis. Non-linear normal modes, part I: a useful framework for the structural dynamicist. Mechanical Systems and Signal Processing, 23(1):170-194, 2009.

[7] D. Laxalde and F. Thouverez. Complex non-linear modal analysis for mechanical systems: Application to turbomachinery bladings with friction interface. Journal of Sound and Vibration, 322(4-5):1009-1025, 2009.

[8] S. W. Shaw and C. Pierre. Normal modes for non-linear vibratory systems. Journal of Sound and Vibration, 164(1):85-124, 1993.

[9] A. H. Nayfeh and S. A. Nayfeh. On nonlinear normal modes of continuous systems. Trans. ASME/Journal of Vibration and Acoustics, 116:129-136, 1994.

[10] A. H. Nayfeh. Reduced-order models of weakly non-linear spatially continuous systems. Nonlinear Dynamics, 16:105-125, 1998. 
[11] E. Pesheck. Reduced-order modeling of nonlinear structural systems using nonlinear normal modes and invariant manifolds. PhD thesis, University of Michigan, 2000.

[12] G. Rega, W. Lacarbonara, and A. H. Nayfeh. Reduction methods for nonlinear vibrations of spatially continuous systems with initial curvature. Solid Mechanics and its applications, 77:235-246, 2000.

[13] C. Touzé, O. Thomas, and A. Chaigne. Hardening/softening behaviour in non-linear oscillations of structural systems using non-linear normal modes. Journal of Sound and Vibration, 273(1-2):77-101, 2004.

[14] C. Touzé, O. Thomas, and A. Huberdeau. Asymptotic non-linear normal modes for large amplitude vibrations of continuous structures. Computers and Structures, 82(3132):2671-2682, 2004.

[15] S.C. Sinha, S. Redkar, and E.A. Butcher. Order reduction of nonlinear systems with time periodic coefficients using invariant manifolds. Journal of Sound and Vibration, 284(35):985-1002, 2005.

[16] M. Amabili and C. Touzé. Reduced-order models for non-linear vibrations of fluid-filled circular cylindrical shells: comparison of pod and asymptotic non-linear normal modes methods. Journal of Fluids and Structures, 23(6):885-903, 2007.

[17] C. Touzé, M. Amabili, and O. Thomas. Reduced-order models for large-amplitude vibrations of shells including in-plane inertia. Computer Methods in Applied Mechanics and Engineering, 197(21-24):2030-2045, 2008.

[18] D. Noreland, S. Bellizzi, C. Vergez, and R. Bouc. Nonlinear modes of clarinet-like musical instruments. Journal of Sound and Vibration, 324(3-5):983-1002, 2009.

[19] A.F. Vakakis, L.I. Manevitch, O. Gendelman, and L. Bergman. Dynamics of linear discrete systems connected to local, essentially non-linear attachments. Journal of Sound and Vibration, 264:559-577, 2003.

[20] G. Kerschen, A.F. Vakakis, Y.S. Lee, D.M. McFarland, J.J. Kowtko, and L.A. Bergman. Energy transfers in a system of two coupled oscillators with essential nonlinearity: 1:1 resonance manifold and transient bridging orbits. Nonlinear Dynamics, 42:283-303, 2005.

[21] E. Gourdon and C.H. Lamarque. Energy pumping for a larger span of energy. Journal of Sound and Vibration, 285:711-720, 2005.

[22] A.F. Vakakis, O.V. Gendelman, L.A. Bergman, D.M. McFarland, G. Kerschen, and Y.S. Lee. Nonlinear targeted energy transfer in mechanical and structural systems I. Springer, New-York, 2008.

[23] A.F. Vakakis, O.V. Gendelman, L.A. Bergman, D.M. McFarland, G. Kerschen, and Y.S. Lee. Nonlinear targeted energy transfer in mechanical and structural systems II. Springer, New-York, 2008.

[24] A.M. Lyapunov. Problème général de la stabilité du mouvement. Annales de la faculté des sciences de Toulouse, Série 2,9:203-474, 1907. 
[25] L.I. Manevitch and Yu.V. Mikhlin. On periodic solutions close to rectilinear normal vibration modes. J. Appl. Math. Mech. (PMM), 36(6):1051-1058, 1972.

[26] R.H. Rand. A direct method for non-linear normal modes. International Journal of Nonlinear Mechanics, 9:363-368, 1974.

[27] M.E. King and A.F. Vakakis. Energy-based formulation for computing nonlinear normal modes in undamped continuous systems. Journal of Vibration and Acoustics, 116:332340, 1994.

[28] Yu.V. Mikhlin. Normal vibrations of a general class of conservative oscillators. Non-linear Dynamics, 11:1-15, 1996.

[29] S. W. Shaw and C. Pierre. Non-linear normal modes and invariant manifolds. Journal of Sound and Vibration, 150(1):170-173, 1991.

[30] J. Carr. Applications of centre manifold theory. Springer-Verlag, New-York, 1981.

[31] H. Poincaré. Les méthodes nouvelles de la mécanique céleste. Gauthiers-Villars, Paris, 1892.

[32] H. Dulac. Solutions d'un système d'équations différentielles dans le voisinage de valeurs singulières. Bulletin de la Société Mathématique de France, 40:324-383, 1912.

[33] L. Jézéquel and C. H. Lamarque. Analysis of non-linear dynamical systems by the normal form theory. Journal of Sound and Vibration, 149(3):429-459, 1991.

[34] C. Touzé and M. Amabili. Non-linear normal modes for damped geometrically non-linear systems: application to reduced-order modeling of harmonically forced structures. Journal of Sound and Vibration, 298(4-5):958-981, 2006.

[35] A.F. Vakakis. Non-linear normal modes (nnms) and their application in vibration theory: an overview. Mechanical Systems and Signal Processing, 11(1):3-22, 1997.

[36] X. Zhang. Geodesics, nonlinear normal modes of conservative vibratory systems and decomposition method. Journal of Sound and Vibration, 282:971-989, 2005.

[37] V.N. Pilipchuk. The calculation of strongly nonlinear systems close to vibration-impact systems. PMM, 49(5):572-578, 1985.

[38] V.N. Pilipchuk. A transformation for vibrating systems based on a non-smooth periodic pair of functions. Dokl. An. Ukr. SSR, 4:37-40, 1988. (in Russian).

[39] V.N. Pilipchuk, A.F. Vakakis, and M.A.F. Azeez. Study of a class of subharmonic motions using a non-smooth temporal transformation (nstt). Physica D, 100:145-164, 1997.

[40] Y.S. Lee, G. Kerschen, A.F. Vakakis, P. Panagopoulos, L. Bergman, and D.M. McFarland. Complicated dynamics of a linear oscillator with a light, essentially non-linear attachment. Physica D, 204:41-69, 2005.

[41] J.C. Slater. A numerical method for determining nonlinear normal modes. Nonlinear Dynamics, 10(1):19-30, 1996. 
[42] R. Seydel. Practical bifurcation and stability analysis. Springer, New-York, 2010. Third edition.

[43] R. Arquier, S. Bellizzi, R. Bouc, and B. Cochelin. Two methods for the computation of nonlinear modes of vibrating systems at large amplitudes. Computers and Structures, 84(24-25):1565-1576, 2006.

[44] M. Peeters, R. Viguié, G. Sérandour, G. Kerschen, and J.C. Golinval. Non-linear normal modes, part II: toward a practical computation using numerical continuation techniques. Mechanical Systems and Signal Processing, 23(1):195-216, 2009.

[45] E.J. Doedel, R. Paffenroth, A.R. Champneys, T.F. Fairgrieve, Y.A. Kuznetsov, B.E. Oldeman, B. Sandstede, and X. Wang. Auto 2000: Continuation and bifurcation software for ordinary differential equations. Technical report, Concordia University, 2002. available at http://cmvl.cs. concordia.ca/auto/.

[46] B. Cochelin and C. Vergez. A high-order purely frequency-based harmonic balance formulation for continuation of periodic solutions. Journal of Sound and Vibration, 324(12):243-262, 2009.

[47] S. Karkar, B. Cochelin, C. Vergez, O. Thomas, and A. Lazarus. User guide manlab 2.0. Technical report, Laboratoire de Mécanique et d'Acoustique (LMA), CNRS UPR 7051, 2002. available at http://manlab.lma.cnrs-mrs.fr/.

[48] A. Dhooge, W. Govaerts, and Y.A. Kuznetsov. Matcont: a matlab package for numerical bifurcation analysis of odes. ACM TOMS, 29(2):141-164, 2003. available at http://sourceforge.net/projects/matcont/.

[49] S. W. Shaw and C. Pierre. Normal modes of vibration for non-linear continuous systems. Journal of Sound and Vibration, 169(3):85-124, 1994.

[50] E. Pesheck, C. Pierre, and S. Shaw. A new Galerkin-based approach for accurate nonlinear normal modes through invariant manifolds. Journal of Sound and Vibration, 249(5):971-993, 2002.

[51] D. Jiang, C. Pierre, and S. Shaw. Large-amplitude non-linear normal modes of piecewise linear systems. Journal of Sound and Vibration, 272(3-5):869-891, 2004.

[52] D. Jiang, C. Pierre, and S. Shaw. Nonlinear normal modes for vibratory systems under harmonic excitation. Journal of Sound and Vibration, 288(4-5):791-812, 2005.

[53] D. Jiang, C. Pierre, and S. Shaw. The construction of non-linear normal modes for systems with internal resonance. International Journal of Non-linear Mechanics, 40(5):729-746, 2005.

[54] M. Peeters, L. Renson, G. Kerschen, J.-C. Golinval, C. Stéphan, and P. Lubrina. Computation of nonlinear normal modes of large structures: application to a full-scale aircraft. In Proc. of the seventh Nonlinear Dynamics conference, ENOC-2011, Roma, 2011.

[55] J. Guckenheimer and P. Holmes. Nonlinear oscillations, dynamical systems and bifurcations of vector fields. Springer-Verlag, New-York, 1983. 
[56] J.-L. Lions. Optimal control of systems governed by partial differential equations. Springer-Verlag, 1971.

[57] C.-H. Lamarque, C. Touzé, and O. Thomas. An upper bound for validity limits of asymptotic analytical approaches based on normal form theory. Non-linear Dynamics, submitted, 2012.

[58] F. Georgiades, M. Peeters, G. Kerschen, J.C. Golinval, and M. Ruzzene. Modal analysis of a nonlinear periodic structure with cyclic symmetry. AIAA Journal, 47:1014-1025, 2009. 